\title{
Perturbed soliton excitations in DNA double helix
}

\author{
M. Daniel ${ }^{a, b} *$, V. Vasumathi ${ }^{a, b}$ \\ a. Centre for Nonlinear Dynamics, Department of Physics, Bharathidasan \\ University, Tiruchirappalli - 620 024, India. \\ b. The Abdus Salam International Centre for Theoretical Physics, Strada \\ Costiera-11, 34014 Trieste, Italy
}

\begin{abstract}
We study nonlinear dynamics of inhomogeneous DNA double helical chain under dynamic plane-base rotator model by considering angular rotation of bases in a plane normal to the helical axis. The DNA dynamics in this case is found to be governed by a perturbed sine-Gordon equation while taking into account the interstrand hydrogen bonding energy between bases and the intrastrand inhomogeneous stacking energy and by making an analogy with the Heisenberg model of the Hamiltonian of an inhomogeneous anisotropic spin ladder with ferromagnetic legs and antiferromagnetic rung coupling. In the homogeneous limit the dynamics is governed by the kink-antikink soliton of the sine-Gordon equation which represents the formation of open state configuration in DNA double helix. The effect of inhomogeneity in stacking energy in the form of localized and periodic variations on the formation of open states in DNA is studied under perturbation. The perturbed soliton is obtained using a multiple scale soliton perturbation theory by solving the associated linear eigen value problem and by constructing the complete set of eigen functions. The inhomogeneity in stacking energy is found to modulate the width and speed of the soliton depending on the nature of inhomogeneity. Also it introduces fluctuations in the form of train of pulses or periodic oscillations in the open state configuration.
\end{abstract}

Key words:

Soliton, DNA, Multiple Scale Perturbation .

$P A C S$ :

87.10.+e, 87.15.He, 66.90.+r, 63.20.Ry

* Corresponding Author. Fax:+91-431-2407093

Email address: daniel@cnld.bdu.ac.in (M. Daniel $\left.{ }^{a, b}\right)$.

Preprint submitted to Elsevier Science 


\section{Introduction}

A number of theoretical models have been proposed to describe nonlinear molecular excitations in DNA double helix which plays an important role in the conservation and transformation of genetic information in biological systems [1]. These theoretical models are based on longitudinal and transverse motions, as well as bending, stretching and rotations [2,3]. Among the different motions, the rotational motion of bases in DNA is found to contribute more towards the opening of base pairs. The first contribution towards nonlinear dynamics of DNA was made by Englander and his co-workers [4] who studied the dynamics of DNA open states by taking into account only the rotational motion of nitrogenous bases, which made the main contribution towards the formation of open states. Yomosa $[5,6]$ developing this idea further proposed a dynamic plane base rotator model which is a generalized version of the Frenkel-Kontrova [7] model that was later improved by Takeno and Homma $[8,9]$ in which attention was paid to the degree of freedom, characterizing base rotations in the plane perpendicular to the helical axis around the backbone structure. In the above, the DNA dynamics was governed by the completely integrable sine-Gordon model admitting kink-type solitons. Then Peyrard and Bishop [10] studied the process of denaturation in which only the transverse motion of bases along the hydrogen bond was taken into account. There was one more model studied by Christiansen and his colleagues (see for e.g.[11]) using Toda lattice model in which two types of internal motions namely, transverse motion along the hydrogen bond direction and longitudinal motion along the backbone direction were found to contribute to DNA denaturation process in terms of travelling solitary waves and standing waves. These localized nonlinear excitations further explain conformation transition $[12,13,14]$, long range interaction of kink solitons in the double chain $[15,16]$, regulation of transcription [15,17], denaturation [10] and charge transport in terms of polarons and bubbles [18]. Some of them have been successfully used for interpreting experimental data related to microwave absorption $[19,20]$. Further developments, in this approach for several years was limited to small improvements of the models involving only numerical methods of simulation of the internal dynamics of DNA $[21,22,23]$. Also, bubbles [24] discrete breathers $[25,26,27]$ and non-breathing compacton-like modes were obtained by solving the DNA-lattice model [28]. Eventhough, the rotation of bases in DNA is mainly due to thermal forces the thermal fluctuation in DNA dynamics has been introduced through random forces in the recent past. For instance, Yakushevich et al [23] has shown that topological solitons of the DNA chains are stable with respect to thermal oscillations. Since random thermal forces introduce only small fluctuations, it is not included in the present study. Thus, the study of nonlinear excitations in DNA molecular chain has become an important task since it is related to its major functions. 
In all the above studies, DNA double helix with homogeneous stiff strands has been considered for the analysis. However, in nature, the presence of different sites along the strands such as promotor, coding, terminator etc. each of which having a very specific sequence of bases and particular functions makes the strands site-dependent or inhomogeneous(soft). Also, defects caused due to external molecules in the sequence and the presence of abasic site-like nonpolar mimic of thymine lead to inhomogeneity $[29,30]$. When included, the DNA dynamics is governed by an inhomogeneous perturbed sine-Gordon equation and thus the problem boils down to solving the same and finding perturbed solitons. Also, in a different context, in the recent times, the study of wave propagation, especially solitons through inhomogeneous or disordered media assumed lot of interest [31]. For instance, kink-impurity interaction and its scattering in the sine-Gordon model was studied in detail by Zhang Fei et al [32]. With this in mind, in the present paper we study the nonlinear dynamics of DNA double helix with inhomogeneous strands by considering a plane base rotator model along the lines of Yomosa. The paper is organized as follows. In section 2 we introduce a dynamic plane base rotator model for the sitedependent DNA double helix and derive the nonlinear dynamical equation. In section 3, we study the effect of inhomogeneity in stacking energy on the open state of DNA in terms of kink-antikink solitons by solving a perturbed sine-Gordon equation using a multiple scale soliton perturbation theory. The results are concluded in section 4. Detailed evaluation of few integrals using residue theorem is given in Appendix.

\section{Plane-base rotator Model and Dynamical equation}

In Fig.1a we have presented a schematic structure of the B-form DNA double helix. Here $S$ and $S^{\prime}$ represent the two complementary strands in the DNA double helix. Each arrow in the figure represents the direction of the base attached to the strand and the dots between arrows represent the net hydrogen bonding effect between the complement bases. While a horizontal projection of the $n^{\text {th }}$ base pair in the XY-plane is represented in Fig.1b, in Fig.1c, we have given the projection of the same in the XZ-plane. The Z-axis is chosen along the helical axis of the DNA. In Fig.1b, $Q_{n}$ and $Q_{n}^{\prime}$ denote the tip of the $n^{\text {th }}$ bases belonging to the complementary strands $S$ and $S^{\prime} . P_{n}$ and $P_{n}^{\prime}$ represent the points where the bases in the $n^{\text {th }}$ base pair are attached to the strands $S$ and $S^{\prime}$ respectively. Let $\left(\theta_{n}, \phi_{n}\right)$ and $\left(\theta_{n}^{\prime}, \phi_{n}^{\prime}\right)$ represent the angles of rotation of the bases in the $n^{\text {th }}$ base pair around the points $P_{n}$ and $P_{n}^{\prime}$ in the $\mathrm{XZ}$ and XY-planes respectively.

The conformation and stability of DNA double helix is mainly determined by the stacking energy between the intrastrand adjacent bases, the hydrogen bonding energy between the interstrand complementary bases and other ener- 


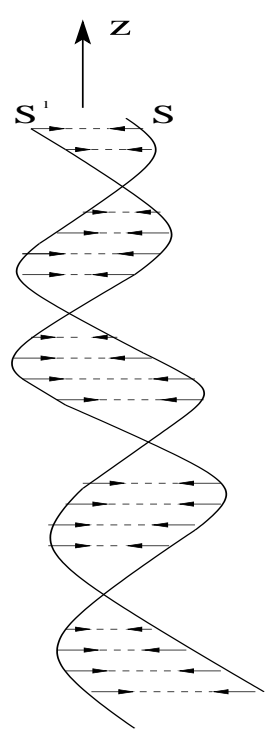

(a)

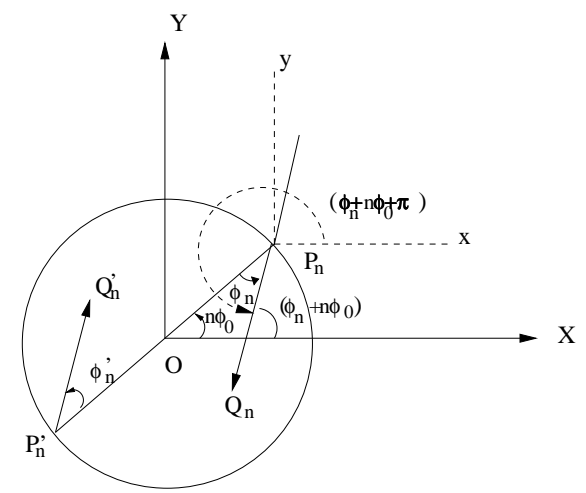

(b)

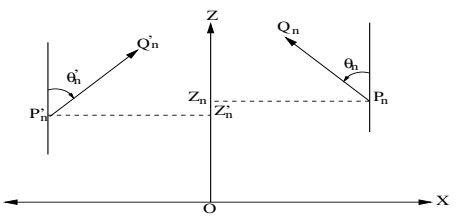

(c)

Fig. 1. (a) A schematic structure B-form DNA double helix. (b) A horizontal projection of the $n^{\text {th }}$ base pair in the XY-plane. (c) A projection of the $n^{\text {th }}$ base pair in the XZ-plane.

gies. From a heuristic argument it was assumed that the interstrand base-base interaction or hydrogen bonding energy of the given base pair depends on the distance between them. Thus, from Fig.1b we can write down the square of the distance between the edges of the arrows $\left(Q_{n} Q_{n}^{\prime}\right)^{2}$ as

$$
\begin{aligned}
\left(Q_{n} Q_{n}^{\prime}\right)^{2}= & 2+4 r^{2}+\left(z_{n}-z_{n}^{\prime}\right)^{2}+2\left(z_{n}-z_{n}^{\prime}\right)\left(\cos \theta_{n}-\cos \theta_{n}^{\prime}\right) \\
& -4 r\left[\sin \theta_{n} \cos \phi_{n}+\sin \theta_{n}^{\prime} \cos \phi_{n}^{\prime}\right]+2\left[\sin \theta_{n} \sin \theta_{n}^{\prime}\right. \\
& \left.\times\left(\cos \phi_{n} \cos \phi_{n}^{\prime}+\sin \phi_{n} \sin \phi_{n}^{\prime}\right)-\cos \theta_{n} \cos \theta_{n}^{\prime}\right]
\end{aligned}
$$

where ' $r$ ' is the radius of the circle depicted in Fig.1b. The base-base interaction energy can be understood in a more clear and transparent way by introducing quasi-spin operators $\mathbf{S}_{\mathbf{n}}=\left(S_{n}^{x}, S_{n}^{y}, S_{n}^{z}\right)$ and $\mathbf{S}_{\mathbf{n}}^{\prime}=\left(S_{n}^{\prime x}, S_{n}^{\prime y}, S_{n}^{\prime z}\right)$ in the form

$$
\begin{gathered}
S_{n}^{x}=\sin \theta_{n} \cos \phi_{n}, \quad S_{n}^{y}=\sin \theta_{n} \sin \phi_{n}, \quad S_{n}^{z}=\cos \theta_{n} \\
S_{n}^{\prime x}=\sin \theta_{n}^{\prime} \cos \phi_{n}^{\prime}, \quad S_{n}^{\prime y}=\sin \theta_{n}^{\prime} \sin \phi_{n}^{\prime}, \quad S_{n}^{\prime z}=\cos \theta_{n}^{\prime}
\end{gathered}
$$

for the $n^{t h}$ bases in the $S^{t h}$ and $S^{\prime}$ th strands respectively. In view of this, Eq. (1) can be written in terms of the given spin operators as follows. 


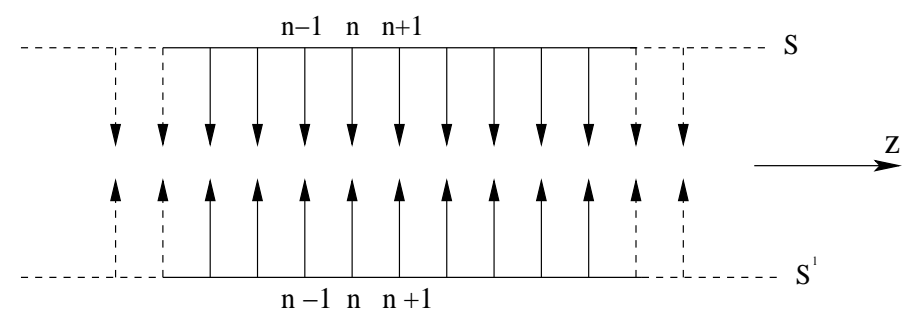

Fig. 2. A schematic representation of DNA as an anisotropic coupled spin chain model or spin ladder.

$$
\left(Q_{n} Q_{n}^{\prime}\right)^{2}=2+4 r^{2}+2\left[S_{n}^{x} S_{n}^{\prime x}+S_{n}^{y} S_{n}^{\prime y}-S_{n}^{z} S_{n}^{\prime z}\right]-4 r\left[S_{n}^{x}+S_{n}^{\prime x}\right]
$$

While writing Eq.(3) we have neglected the longitudinal compression along the direction of the helical axis thereby choosing $z_{n}=z_{n}^{\prime}$. It may be noted that the form of $\left(Q_{n} Q_{n}^{\prime}\right)^{2}$ given in Eq.(3) is the same as the Hamiltonian for a generalized form of the Heisenberg spin model. Therefore, the intrastrand base-base interaction in DNA can be written using the same consideration. It is known that stacking or base-base interaction is a dominant force that stabilizes the DNA double helix. It is much stronger than the hydrogen bonding force and in fact the stacking between adjacent bases often contributes more than half of the total free energy of the base pairs [33]. Several non-covalent forces including dipole-dipole interaction, van der Waals force etc stabilize stacking in DNA. It is also reasonable to think that if such a quasi-spin model can be used in this problem, the double strand DNA and the rung-like base pairs can be conceived as an anisotropic coupled spin chain model or spin ladder. This is schematically presented in Fig.2. In the figure, $S$ and $S^{\prime}$ which previously represented two strands of the DNA here correspond to two ferromagnetic lattices of a spin ladder with antiferromagnetic coupling among the rungs. In the case of spin chains each arrow represents the magnetic moment corresponding to a group of atoms at that lattice point. Due to antiferromagnetic type of rung coupling the arrows in the lattice $S$ and $S^{\prime}$ are marked anti-parallel to each other. Here Z-direction (i.e) the direction of the helical axis is chosen as the easy axis of magnetization in the spin chain. Further, the spin-spin exchange interaction is restricted. to the nearest neighbours, (i.e) the $n^{\text {th }}$ spin is coupled to the spins at the $(n+1)^{t h}$ and $(n-1)^{t h}$ sites.

With this consideration we use the following Heisenberg model of the Hamiltonian for an anisotropic coupled spin chain model or spin ladder with sitedependent or inhomogeneous ferromagnetic-type exchange interaction between nearest neighbouring spins in the same lattice (equivalent to coupling among bases in the same strand i.e. intrastrand interaction) and antiferromagnetic rung-coupling (between bases belonging to the complementary strands i.e. interstrand interaction). 


$$
\begin{aligned}
H= & \sum_{n}\left[-J f_{n}\left(S_{n}^{x} S_{n+1}^{x}+S_{n}^{y} S_{n+1}^{y}\right)-K f_{n} S_{n}^{z} S_{n+1}^{z}-J^{\prime} f_{n}^{\prime}\left(S_{n}^{\prime x} S_{n+1}^{\prime x}\right.\right. \\
& \left.+S_{n}^{\prime y} S_{n+1}^{\prime y}\right)-K^{\prime} f_{n}^{\prime} S_{n}^{\prime z} S_{n+1}^{\prime z}+\eta\left(S_{n}^{x} S_{n}^{\prime x}+S_{n}^{y} S_{n}^{\prime y}\right)+\mu S_{n}^{z} S_{n}^{\prime z} \\
& \left.+A\left(S_{n}^{z}\right)^{2}+A^{\prime}\left(S_{n}^{\prime z}\right)^{2}\right] .
\end{aligned}
$$

In the above Hamiltonian $J$ and $J^{\prime}$ correspond to the intrastrand interaction constant or the stacking energy between the $n^{\text {th }}$ base and its nearest neighbours in the plane normal to the helical axis in the strands $S$ and $S^{\prime}$ respectively. When $\mathrm{K}$ and $K^{\prime}$ are not equal to $J$ and $J^{\prime}$ respectively, they introduce anisotropy in the intrastrand interaction. $\mu$ and $\eta$ represent a measure of interstrand interaction or hydrogen bonding energy between the bases of similar sites in both the strands along the direction of the helical axis and in a plane normal to it respectively. Here we have assumed that there exist on an average almost uniform interactions $A$ and $A^{\prime}$ that assume positive values which are the uniaxial anisotropy coefficients leading to rotation of the bases in a plane normal to the helical axis. The quantities $f_{n}$ and $f_{n}^{\prime}$ in Hamiltonian (4) indicate that the intrastrand stacking energy between bases in the $S^{t h}$ and $S^{\prime t h}$ strand varies in a specified site dependent fashion which leads to inhomogeneity in the DNA double helical chain. In general $f_{n}$ and $f_{n}^{\prime}$ may take different values for different base sequences. The inhomogeneity in DNA double helix may arise due to any one of the reasons mentioned in the previous section. The effect of inhomogeneity in DNA nonlinear dynamics has been studied in the past by several authors in various contexts such as variational mass density of bases [34] and defect [25].

To proceed further we rewrite Hamiltonian (4) in terms of the variables $\left(\theta_{n}, \phi_{n}\right)$ and $\left(\theta_{n}^{\prime}, \phi_{n}^{\prime}\right)$ using Eqs.(2) and obtain

$$
\begin{aligned}
H= & \sum_{n}\left[-J f_{n} \sin \theta_{n} \sin \theta_{n+1} \cos \left(\phi_{n+1}-\phi_{n}\right)-K f_{n} \cos \theta_{n} \cos \theta_{n+1}\right. \\
& -J^{\prime} f_{n}^{\prime} \sin \theta_{n}^{\prime} \sin \theta_{n+1}^{\prime} \cos \left(\phi_{n+1}^{\prime}-\phi_{n}^{\prime}\right)-K^{\prime} f_{n}^{\prime} \cos \theta_{n}^{\prime} \cos \theta_{n+1}^{\prime} \\
& +\eta \sin \theta_{n} \sin \theta_{n}^{\prime} \cos \left(\phi_{n}-\phi_{n}^{\prime}\right)+\mu \cos \theta_{n} \cos \theta_{n}^{\prime}+A \cos \theta_{n}^{2} \\
& \left.+A^{\prime} \cos \theta_{n}^{\prime 2}\right] .
\end{aligned}
$$

The quasi-spin model thus introduced implies that the dynamics of bases in DNA can be described by the following equations of motion.

$$
\dot{\theta}_{n}=\frac{1}{\sin \theta_{n}} \frac{\partial H}{\partial \phi_{n}}, \dot{\phi}_{n}=\frac{-1}{\sin \theta_{n}} \frac{\partial H}{\partial \theta_{n}}, \dot{\theta}_{n}^{\prime}=\frac{1}{\sin \theta_{n}^{\prime}} \frac{\partial H}{\partial \phi_{n}^{\prime}}, \quad \dot{\phi}_{n}^{\prime}=\frac{-1}{\sin \theta_{n}^{\prime}} \frac{\partial H}{\partial \theta_{n}^{\prime}} .
$$

In Eq.(6) the overdot represents time derivative. When the anisotropy energies $A$ and $A^{\prime}$ are much larger than the other interactions, (i.e) when $A, A^{\prime}>>$ 
$J, J^{\prime}, K, K^{\prime}, \eta, \mu$, then the equations of motion (6) on substituting the Hamiltonian (5) become

$$
\dot{\phi}_{n}=2 A \cos \theta_{n}, \quad \dot{\phi}_{n}^{\prime}=2 A^{\prime} \cos \theta_{n}^{\prime}
$$

The other two equations in (6) satisfy identically. Using Eq. (7) in the Hamiltonian (5) we obtain

$$
\begin{aligned}
H= & \sum_{n}\left[\frac{I}{2} \dot{\phi}_{n}^{2}+\frac{I^{\prime}}{2} \dot{\phi}_{n}^{\prime 2}-J f_{n} \sin \theta_{n} \sin \theta_{n+1} \cos \left(\phi_{n+1}-\phi_{n}\right)-K f_{n} \cos \theta_{n}\right. \\
& \times \cos \theta_{n+1}-J^{\prime} f_{n}^{\prime} \sin \theta_{n}^{\prime} \sin \theta_{n+1}^{\prime} \cos \left(\phi_{n+1}^{\prime}-\phi_{n}^{\prime}\right)-K^{\prime} f_{n}^{\prime} \cos \theta_{n}^{\prime} \cos \theta_{n+1}^{\prime} \\
& \left.+\eta \sin \theta_{n} \sin \theta_{n}^{\prime} \cos \left(\phi_{n}-\phi_{n}^{\prime}\right)+\mu \cos \theta_{n} \cos \theta_{n}^{\prime}\right]
\end{aligned}
$$

where $I=\frac{1}{2 A}$ and $I^{\prime}=\frac{1}{2 A^{\prime}}$. The above Hamiltonian can be rewritten in the limits of plane-base rotator $\left(\theta_{n}=\theta_{n}^{\prime}=\pi / 2\right)$ and absolute minima of potential as

$$
\begin{aligned}
H= & \sum_{n}\left[\frac{I}{2} \dot{\phi}_{n}^{2}+\frac{I^{\prime}}{2} \dot{\phi}_{n}^{\prime 2}+J f_{n}\left[1-\cos \left(\phi_{n+1}-\phi_{n}\right)\right]\right. \\
& \left.+J^{\prime} f_{n}^{\prime}\left[1-\cos \left(\phi_{n+1}^{\prime}-\phi_{n}^{\prime}\right)\right]-\eta\left[1-\cos \left(\phi_{n}-\phi_{n}^{\prime}\right)\right]\right] .
\end{aligned}
$$

It may be noted that the above limit corresponds to the XY-spin model of two coupled inhomogeneous ferromagnetic spin system. In Hamiltonian (9) the first two terms represent the kinetic energies of the rotational motion of the $n^{\text {th }}$ nucleotide bases accompanied by the potential energy associated with the $n^{\text {th }}$ nucleotide sugar and phosphate and its complementary unit around the axes at $P_{n}$ and $P_{n}^{\prime}$ (see Fig.1b). $I$ and $I^{\prime}$ are the moments of inertia of the nucleotides around the axes at $P_{n}$ and $P_{n}^{\prime}$ respectively. It may be further noted that in the new Hamiltonian the term proportional to $\mu$ vanishes. Now, using Hamiltonian (9), the Hamilton's equations of motion can be immediately written as

$$
\begin{aligned}
I \ddot{\phi}_{n} & =J\left[f_{n} \sin \left(\phi_{n+1}-\phi_{n}\right)-f_{n-1} \sin \left(\phi_{n}-\phi_{n-1}\right)\right]+\eta \sin \left(\phi_{n}-\phi_{n}^{\prime}\right),(10 \\
I^{\prime} \ddot{\phi}_{n}^{\prime} & =J^{\prime}\left[f_{n}^{\prime} \sin \left(\phi_{n+1}^{\prime}-\phi_{n}^{\prime}\right)-f_{n-1}^{\prime} \sin \left(\phi_{n}^{\prime}-\phi_{n-1}^{\prime}\right)\right]+\eta \sin \left(\phi_{n}^{\prime}-\phi_{n}\right) .(10
\end{aligned}
$$

Eqs.(10a) and (10b) describe the dynamics of DNA in a plane-base rotator model at the discrete level while considering the dominant angular rotation of the bases in a plane normal to the helical axis and ignoring all other small motions of the bases.

It is expected that in the B-form of DNA double helix the difference in the angular rotation of bases with respect to neighbouring bases along the two 
strands is small $[8,9]$. Hence we assume that $\sin \left(\phi_{n \pm 1}-\phi_{n}\right) \approx\left(\phi_{n \pm 1}-\phi_{n}\right)$ and $\sin \left(\phi_{n \pm 1}^{\prime}-\phi_{n}^{\prime}\right) \approx\left(\phi_{n \pm 1}^{\prime}-\phi_{n}^{\prime}\right)$ in Eqs.(10a, b). Also, as the length of the DNA chain is very large involving several thousands of base pairs compared to the distance between the neighbouring bases along the strands we make a continuum approximation as done by several authors in the past $[2,3,4,5,6,7,8,9,10]$ which is valid in the long wavelength, low temperature limit by introducing two fields of rotational angles, $\phi_{n}(t) \rightarrow \phi(z, t), \phi_{n}^{\prime}(t) \rightarrow \phi^{\prime}(z, t)$ and two inhomogeneous stacking fields, $f_{n} \rightarrow f(z)$ and $f_{n}^{\prime} \rightarrow f^{\prime}(z)$ along with the following expansions.

$$
\phi_{n \pm 1}=\phi(z, t) \pm a \frac{\partial \phi}{\partial z}+\frac{a^{2}}{2 !} \frac{\partial^{2} \phi}{\partial z^{2}} \pm \ldots, f_{n \pm 1}=f(z) \pm a \frac{\partial f}{\partial z}+\frac{a^{2}}{2 !} \frac{\partial^{2} f}{\partial z^{2}} \pm
$$

where ' $a$ ' is the lattice parameter along both the strands $S$ and $S^{\prime}$. In a similar way we write down expansions for $\phi_{n \pm 1}^{\prime}$ and $f_{n \pm 1}^{\prime}$. As the inhomogeneity here is site-dependent and associated with the bases themselves, we have chosen same lattice parameter ' $a$ ' for both $\phi_{n \pm 1}$ and $f_{n \pm 1}$. Under this continuum approximation the equations of motion $(10 \mathrm{a}, \mathrm{b})$ upto $\mathrm{O}\left(a^{2}\right)$ is written as

$$
\begin{aligned}
I \phi_{t t} & =J a^{2}\left[f(z) \phi_{z z}+f_{z} \phi_{z}\right]+\eta \sin \left(\phi-\phi^{\prime}\right), \\
I^{\prime} \phi_{t t}^{\prime} & =J^{\prime} a^{2}\left[f^{\prime}(z) \phi_{z z}^{\prime}+f_{z}^{\prime} \phi_{z}^{\prime}\right]+\eta \sin \left(\phi^{\prime}-\phi\right) .
\end{aligned}
$$

In Eqs. (12) the suffices $t$ and $z$ represent partial derivatives with respect to time $t$ and the spatial variable $z$ respectively.

In a DNA chain, the two strands are expected to exhibit similar type of macroscopic physical behaviour and hence we assume that $I=I^{\prime}, J=J^{\prime}$ and $f=f^{\prime}$. In view of this, Eqs. (12) after rescaling the time variable as $\hat{t}=\sqrt{\frac{J a^{2}}{I}} t$ and choosing $\eta=-\frac{J a^{2}}{2}$ for future convenience can be written as

$$
\begin{aligned}
& \phi_{\hat{t} \hat{t}}=\left[f(z) \phi_{z z}+f_{z} \phi_{z}\right]-\frac{1}{2} \sin \left(\phi-\phi^{\prime}\right), \\
& \phi_{\hat{t} \hat{t}}^{\prime}=\left[f(z) \phi_{z z}^{\prime}+f_{z} \phi_{z}^{\prime}\right]-\frac{1}{2} \sin \left(\phi^{\prime}-\phi\right) .
\end{aligned}
$$

It is more convenient to describe the transverse motion of bases in DNA strands in terms of the centre of mass co-ordinates. For this, we rewrite Eqs. (13a) and (13b) by subtracting and adding them respectively.

$$
\begin{aligned}
& \left(\phi-\phi^{\prime}\right)_{\hat{t} \hat{t}}=f(z)\left(\phi-\phi^{\prime}\right)_{z z}+f_{z}\left(\phi-\phi^{\prime}\right)_{z}-\sin \left(\phi-\phi^{\prime}\right) \\
& \left(\phi+\phi^{\prime}\right)_{\hat{t} \hat{t}}=f(z)\left(\phi+\phi^{\prime}\right)_{z z}+f_{z}\left(\phi+\phi^{\prime}\right)_{z}
\end{aligned}
$$


In a different context while studying the magnetoelastic effect induced by interaction between two ferromagnetically coupled XY-spin chains in the static limit Dandoloff and Saxena [35] obtained similar equations. To commence the open state configuration in DNA, the two complementary bases are expected to rotate in opposite directions so that $\phi=-\phi^{\prime}$, and in this case Eq. (14b) satisfies identically and Eq. (14a) becomes

$$
\Psi_{\hat{t} \hat{t}}=f \Psi_{z z}+f_{z} \Psi_{z}-\sin \Psi
$$

where $\Psi=2 \phi$. Assuming small inhomogeneity along the strands by choosing $f(z)=1+\epsilon g(z)$ where $\epsilon$ is a small parameter and $g(z)$ a measure of the inhomogeneity, Eq. (15) can be written as

$$
\Psi_{\hat{t} \hat{t}}-\Psi_{z z}+\sin \Psi=\epsilon\left[g(z) \Psi_{z}\right]_{z}
$$

Eq. (16) describes the dynamics of bases under rotation in a plane-base rotator model of an inhomogeneous DNA double helical chain. When $\epsilon=0$, Eq. (16) reduces to the completely integrable sine-Gordon equation which admits kink and antikink-type of soliton solutions and hence we call Eq.(16) as a perturbed sine-Gordon equation. The integrable sine-Gordon equation ( $\epsilon=0$ case) was originally solved for N-soliton solutions using the most celebrated Inverse Scattering Transform (IST) method by Ablowitz and his co-workers [36]. The kink and antikink soliton solutions of the integrable sine-Gordon equation (Eq.(16) when $\epsilon=0$ ) are depicted in Figs.3a and 3b. The kink-antikink solitons of the sine-Gordon equation describe an open state configuration in the DNA double helix. The formation of open state configuration in terms of kink-antikink pair in DNA double helical chain is schematically represented in Fig.3c. In this figure the base pairs are found to open locally in the form of kink-antikink structure in each strand and propagate along the direction of the helical axis.

\section{Effect of stacking energy Inhomogeneity on the Open State}

\subsection{A Perturbation approach}

When inhomogeneity in stacking is present (i.e) when terms proportional to $\epsilon$ are present in Eq.(16), the inhomogeneity is expected to perturb the kink and antikink solitons corresponding to the open state of DNA. One of the most powerful techniques in dealing with perturbed soliton equations is the soliton perturbation theory which is based on the IST method $[37,38]$. However, as the method is very sophisticated it is very difficult to use the same in several 


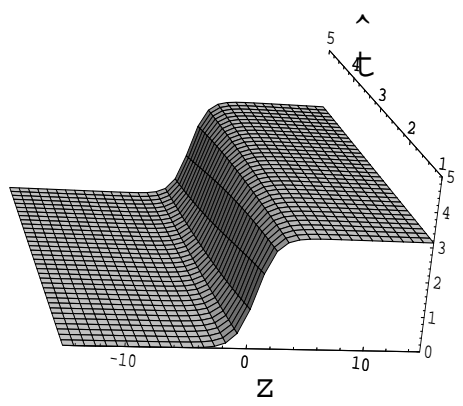

(a)

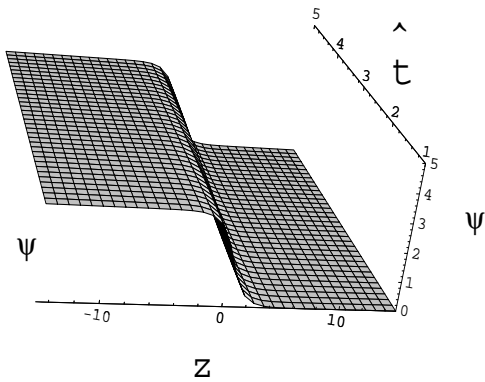

(b)

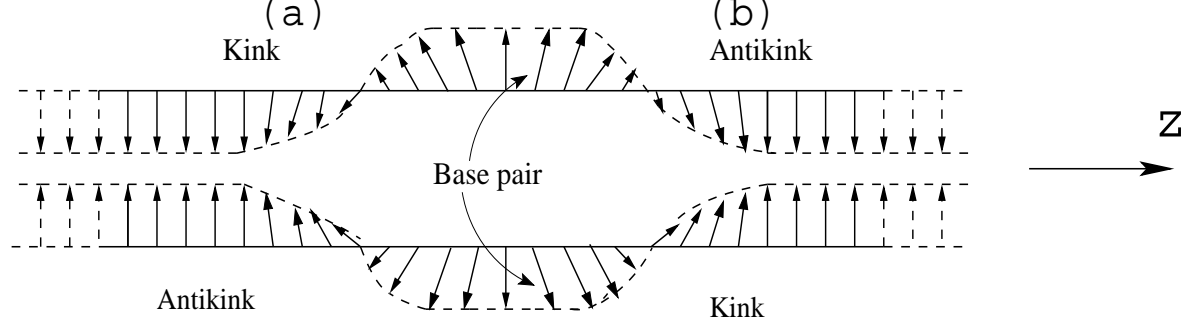

(c)

Fig. 3. (a) Kink and (b) antikink soliton solutions of the sine-Gordon equation (Eq. (16) when $\epsilon=0$ ). (c) A sketch of the formation of open state configuration in terms of kink-antikink solitons in DNA double helix.

cases. In view of this, a direct method to study the soliton perturbation was first introduced by Gorshkov and Ostrovskii [39] and later many authors used different types of direct methods to study soliton perturbation (see for e.g. refs. $[40,41,42,43,44,45])$. The characteristic feature of this method is that the perturbed nonlinear equation is linearized by expanding its solution about the unperturbed solution and the eigen functions for the operator associated with the linearized equation are found out. The complete solution is then written in terms of these eigen functions [46,47]. In these methods the basic fact that the presence of perturbation not only modifies the shape of the soliton by a correction of linear dispersion but also undergoes a slow time change of the soliton parameters have been acknowledged. In this paper, we use one such direct perturbation method which is also dealt in reference [48] in a different context to solve the perturbed sine-Gordon equation (16) and to understand the effect of inhomogeneity in stacking energy on the open state configuration of DNA. The procedure we adapt here is based on the derivative expansion method to linearize the perturbed sine-Gordon equation in the co-ordinate frame attached to the moving frame. The parameters of the kink-antikink soliton are assumed to depend on a slow time scale in order to eliminate the secular terms. The linearized equations will be solved using the method of separation of variables which ultimately will be related to an eigen value problem, the eigen functions of which form the bases of the perturbed 
solution. The eigen functions contain information about the time dependence of the soliton parameters and help to calculate the perturbed soliton. In the following we use the above approach to find the perturbed soliton solution of Eq.(16).

\subsection{Linearization of the perturbed sine-Gordon equation}

When the perturbation is absent (i.e) when $\epsilon=0$ in Eq. (16) the unperturbed integrable sine-Gordon equation provides N-soliton solutions and the one soliton solution (see also Figs.3a and $3 \mathrm{~b}$ is written as

$$
\Psi(z, \hat{t})=4 \arctan \exp [ \pm m(z-v \hat{t})], \quad m=\frac{1}{\sqrt{1-v^{2}}}
$$

In Eq.(17) while the upper sign corresponds to kink soliton, the lower sign represents the antikink soliton. Here $v$ and $m^{-1}$ are real parameters that determine the velocity and width of the soliton respectively. In order to study the effect of perturbation the time variable $\hat{t}$ is transformed into several variables as $t_{n}=\epsilon^{n} \hat{t}$ where $\mathrm{n}=0,1,2, \ldots$ and $\epsilon$ is a very small parameter. In view of this, the time derivative in Eq. (16) is replaced by the expansion

$$
\frac{\partial}{\partial \hat{t}}=\frac{\partial}{\partial t_{0}}+\epsilon \frac{\partial}{\partial t_{1}}+\epsilon^{2} \frac{\partial}{\partial t_{2}}+\ldots
$$

Simultaneously $\Psi$ is expanded in an asymptotic series as

$$
\Psi=\Psi^{(0)}+\epsilon \Psi^{(1)}+\epsilon^{2} \Psi^{(2)}+\ldots
$$

Using the above expansions for $\hat{t}$ and $\Psi$ in Eq. (16) and equating the coefficients of different powers of $\epsilon$, we obtain the following equations.

$$
\begin{array}{ll}
\epsilon^{(0)}: & \Psi_{t_{0} t_{0}}^{(0)}-\Psi_{z z}^{(0)}+\sin \Psi^{(0)}=0 \\
\epsilon^{(1)}: & \Psi_{t_{0} t_{0}}^{(1)}-\Psi_{z z}^{(1)}+\cos \Psi^{(0)} \Psi^{(1)}=g \Psi_{z z}^{(0)}+g_{z} \Psi_{z}^{(0)}-2 \Psi_{t_{0} t_{1}}^{(0)}
\end{array}
$$

etc.

The initial conditions for perturbation in the case of the single soliton given in Eq. (17) is written as

$$
\Psi^{(0)}(z, 0)=4 \arctan \exp m z, \Psi^{(n)}(z, 0)=\Psi_{t_{0}}^{(n)}(z, 0)=0, n=0,1, \ldots
$$


The equation obtained at order of $\epsilon^{(0)}$, (i.e) Eq.(20a) is just the integrable sineGordon equation for $\psi^{(0)}$, the single soliton solution of which can be written from Eq. (17) immediately as

$$
\Psi^{(0)}\left(z, t_{0}\right)=4 \arctan \exp \zeta, \zeta= \pm m_{0}(z-\xi), \xi_{t_{0}}=v_{0}
$$

where $v_{0}$ is the velocity of soliton in the $t_{0}$-time scale. Due to perturbation, the soliton parameters namely $m$ and $\xi$ are now treated as functions of the slow time variables $t_{0}, t_{1}, t_{2}, \ldots$. However $\mathrm{m}$ is treated independent of $t_{0}$. In view of the above, Eq. (20b) becomes

$$
\Psi_{t_{0} t_{0}}^{(1)}-\Psi_{z z}^{(1)}+\left(1-2 \operatorname{sech}^{2} \zeta\right) \Psi^{(1)}=F^{(1)}(z)
$$

where

$$
F^{(1)}(z)=2[g(z) \operatorname{sech} z]_{z}+4 v_{0} \operatorname{sech} z\left[m_{t_{1}}+\left(m^{2} \xi_{t_{1}}-z m_{t_{1}}\right) \tanh z\right]
$$

While writing the above equation we have used the result $\cos \Psi^{(0)}=1-$ $2 \operatorname{sech}^{2} \zeta$ obtained from the solution of the unperturbed equation (20a). In order to represent everything in a co-ordinate system moving with the soliton, we transform $z=\frac{\zeta}{m}+v t_{0}$ and $t_{0}=t_{0}+\zeta$, so that Eqs. (23) and the initial conditions given in Eq. (21a) can be written as

$$
\Psi_{t_{0} t_{0}}^{(1)}-2 m v_{0} \Psi_{t_{0} \zeta}^{(1)}-\Psi_{\zeta \zeta}^{(1)}+\left(1-2 \operatorname{sech}^{2} \zeta\right) \Psi^{(1)}=F^{(1)}\left(\zeta, t_{0}\right)
$$

where

$$
F^{(1)}\left(\zeta, t_{0}\right)=2[g(\zeta) \operatorname{sech} \zeta]_{\zeta}+4 v_{0} \operatorname{sech} \zeta\left[m_{t_{1}}+\left(m^{2} \xi_{t_{1}}-\zeta m_{t_{1}}\right) \tanh \zeta\right](24
$$

and

$$
\Psi^{(1)}(\zeta, 0)=0, \quad \Psi_{t_{0}}^{(1)}(\zeta, 0)=0
$$

We now introduce one more transformation $\tau=\frac{t_{0}}{2 m}-\frac{\left(1+v_{0}\right) \zeta}{2}$ on the independent variable to eliminate the first term in the left hand side of Eq. (24a). Thus on using the above transformation Eq. (24a) becomes

$$
\Psi_{\tau \zeta}^{(1)}-\Psi_{\zeta \zeta}^{(1)}+\left(1-\operatorname{sech}^{2} \zeta\right) \Psi^{(1)}=F^{(1)}(\zeta, \tau)
$$

where $F^{(1)}(\zeta, \tau)$ equals exactly the right hand side of Eq. (24b). The solution of Eq. (25) is searched by assuming 


$$
\Psi^{(1)}(\zeta, \tau)=X(\zeta) T(\tau), \quad F^{(1)}(\zeta, \tau)=X_{\zeta}(\zeta) H(\tau)
$$

Substituting Eq. (26) in Eq. (25) we obtain

$$
\frac{1}{X_{\zeta}}\left[X_{\zeta \zeta}+\left(2 \operatorname{sech}^{2} \zeta-1\right) X\right]=\frac{1}{T}\left[T_{\tau}-H(\tau)\right] .
$$

In Eq. (27) the left hand side is independent of $\tau$ and the right hand side is independent of the variable $\zeta$. Hence we can equate the left and right hand sides of Eq. (27) to a constant say $\lambda_{0}$ and write

$$
\begin{aligned}
X_{\zeta \zeta}+\left(2 \operatorname{sech}^{2} \zeta-1\right) X & =\lambda_{0} X_{\zeta} \\
T_{\tau}-\lambda_{0} T & =H(\tau) .
\end{aligned}
$$

Thus, the problem of constructing the perturbed soliton at this moment turns out to be solving Eqs. (28a) and (28b) by constructing the eigen functions and finding the eigen values. It may be noted that Eq. (28a) is a generalized eigen value problem which is not a self-adjoint eigen value problem and differing from the normal eigen value problem with $X_{\zeta}$ in the right hand side instead of $X$ and Eq. (28b) is a first order linear inhomogeneous differential equation which can be solved using known procedure.

\subsection{Solving the eigen value problem}

Before actually solving the eigen value equation (28a) we first consider it in a more general form given by

$$
L_{1} X=\lambda \tilde{X}, \quad L_{1}=\partial_{\zeta \zeta}+2 \operatorname{sech}^{2} \zeta-1
$$

where $\lambda$ is the eigen value. In order to find the adjoint eigen function to $X$, we consider another eigen value problem

$$
L_{2} \tilde{X}=\lambda X
$$

where the operator $L_{2}$ is still to be determined. The eigen value equations (29) and (30) can be combined to give

$$
L_{2} L_{1} X=\lambda^{2} X, \quad L_{1} L_{2} \tilde{X}=\lambda^{2} \tilde{X}
$$

Since we already know the form of $L_{1}$ as given in Eq.(29), if we choose the operator $L_{2}$ as $L_{2}=\partial_{\zeta \zeta}+6 \operatorname{sech}^{2} \zeta-1$, it can be verified that $L_{1} L_{2}$ is the 
adjoint of $L_{2} L_{1}$. Thus $\mathrm{X}$ and $\tilde{X}$ are expected to be adjoint eigen functions.

Now for solving the eigen value equations (29) and (30), we choose the eigen functions $X$ and $\tilde{X}$ to be in the form

$$
X(\zeta, k)=p(\zeta, k) e^{i k \zeta}, \quad \tilde{X}(\zeta, k)=q(\zeta, k) e^{i k \zeta}
$$

where $p(\zeta, k)$ and $q(\zeta, k)$ are assumed to have the asymptotic behaviour $p(\zeta, k) \rightarrow$ a constant and $q(\zeta, k) \rightarrow$ a constant as $\zeta \rightarrow \pm \infty$ and $k$ is the propagation constant. On using these asymptotic forms for $p(\zeta, k)$ and $q(\zeta, k)$ in Eq. (32) and then substituting the resultant $X(\zeta, k)$ and $\tilde{X}(\zeta, k)$ in Eqs. (29) and (30) we obtain the eigen value as

$$
\lambda=-\left(k^{2}+1\right)
$$

On substituting the exact forms of $X$ and $\tilde{X}$ from Eq. (32) in Eqs. (29) and (30) we get the following set of ordinary differential equations for $p(\zeta, k)$ and $q(\zeta, k)$.

$$
\begin{gathered}
\left(L_{1}-k^{2}\right) p+2 i k p_{\zeta}+\left(1+k^{2}\right) q=0 \\
\left(L_{2}-k^{2}\right) q+2 i k q_{\zeta}+\left(1+k^{2}\right) p=0
\end{gathered}
$$

For solving the above equations we expand $p(\zeta, k)$ and $q(\zeta, k)$ in the following series [48].

$$
\begin{aligned}
& p(\zeta, k)=p_{0}+p_{1} \frac{\sinh \zeta}{\cosh \zeta}+p_{2} \frac{1}{\cosh ^{2} \zeta}+p_{3} \frac{\sinh \zeta}{\cosh ^{3} \zeta}+p_{4} \frac{1}{\cosh ^{4} \zeta}+\ldots, \\
& q(\zeta, k)=q_{0}+q_{1} \frac{\sinh \zeta}{\cosh \zeta}+q_{2} \frac{1}{\cosh ^{2} \zeta}+q_{3} \frac{\sinh \zeta}{\cosh ^{3} \zeta}+q_{4} \frac{1}{\cosh ^{4} \zeta} \ldots
\end{aligned}
$$

where the coefficients $p_{j}$ and $q_{j}, \mathrm{j}=0,1,2, \ldots$ are functions of $k$ which are to be determined. We substitute the series expansions given in Eqs. (35a) and (35b) in Eqs.(34) and collect the coefficients of $1, \frac{\sinh \zeta}{\cosh \zeta}, \frac{1}{\cosh ^{2} \zeta}, \ldots$ and obtain the following algebraic equations.

$$
\begin{gathered}
p_{0}=q_{0}, \quad p_{1}=q_{1} \\
2 p_{0}+2 i k p_{1}+\left(3-k^{2}\right) p_{2}-4 i k p_{3}+\left(1+k^{2}\right) q_{2}=0 \\
6 q_{0}+2 i k q_{1}+\left(3-k^{2}\right) q_{2}-4 i k q_{3}+\left(1+k^{2}\right) p_{2}=0 \\
-4 i k p_{2}+\left(3-k^{2}\right) p_{3}+\left(1+k^{2}\right) q_{3}=0 \\
4 q_{1}-4 i k q_{2}+\left(3-k^{2}\right) q_{3}+\left(1+k^{2}\right) p_{3}=0 \\
\text { etc. }
\end{gathered}
$$


By assuming $p_{j}=q_{j}=0$ for $j \geq 3$, and substituting these values in Eqs.(36d) and (36e), we obtain

$$
p_{2}=0, \quad q_{2}=-i \frac{q_{1}}{k}
$$

On substituting the results given in Eq.(37) in Eqs. (36b) and (36c) we get

$$
p_{1}=q_{1}=-\frac{2 i k p_{0}}{\left(1-k^{2}\right)}, \quad q_{2}=-\frac{2 p_{0}}{\left(1-k^{2}\right)} .
$$

For our convenience we choose $p_{0}=q_{0}=c\left(1-k^{2}\right)$ and on substituting this in the above equations, we obtain the other coefficients as follows.

$$
p_{1}=q_{1}=-2 i k, p_{2}=0, q_{2}=-2 c .
$$

Here ' $c$ ' is an arbitrary constant which will be determined. Using the above values in Eqs. (35a) and (35b), and then in Eq. (32), we finally obtain

$$
\begin{aligned}
& X(\zeta, k)=c\left(1-k^{2}-2 i k \tanh \zeta\right) e^{i k \zeta} \\
& \tilde{X}(\zeta, k)=c\left(1-k^{2}-2 i k \tanh \zeta-2 \operatorname{sech}^{2} \zeta\right) e^{i k \zeta}
\end{aligned}
$$

On comparing Eqs. (40a) and (40b) we can write

$$
\tilde{X}(\zeta, k)=\frac{X_{\zeta}(\zeta, k)}{i k}
$$

Using Eq.(41) in the right hand side of Eq.(29) and comparing the resultant equation with (28a), we can write down the eigen value $\lambda_{0}$ as

$$
\lambda_{0}=i \frac{\left(1+k^{2}\right)}{k} .
$$

Now to determine the constant ' $c$ ' we use the orthonormality relation between $X(\zeta, k)$ and $\tilde{X}(\zeta, k)$ given by

$$
\int_{-\infty}^{\infty} X(\zeta, k) \tilde{X}^{*}\left(\zeta, k^{\prime}\right) d \zeta=\delta\left(k-k^{\prime}\right)
$$

On substituting the eigen functions $X(\zeta, k)$ and $\tilde{X}(\zeta, k)$ given in Eqs. (40a) and (40b) respectively in Eq.(43) and after evaluating the integral we obtain 


$$
2 \pi c^{2}\left[\left(1-k^{2}\right)\left(1-k^{\prime 2}\right)+4 k k^{\prime}\right] \delta\left(k-k^{\prime}\right)=\delta\left(k-k^{\prime}\right),
$$

which gives $c^{2}=\frac{1}{2 \pi}\left(1+k^{2}\right)^{-2}$. The correct form of the eigen functions $X(\zeta, k)$ and $\tilde{X}(\zeta, k)$ is written down after using the above value of ' $c$ ' in Eqs. (40a) and (40b).

$$
\begin{aligned}
& X(\zeta, k)=\frac{\left(1-k^{2}-2 i k \tanh \zeta\right)}{\sqrt{2 \pi}\left(1+k^{2}\right)} e^{i k \zeta} \\
& \tilde{X}(\zeta, k)=\frac{\left(1-k^{2}-2 i k \tanh \zeta-2 \operatorname{sech}^{2} \zeta\right)}{\sqrt{2 \pi}\left(1+k^{2}\right)} e^{i k \zeta}
\end{aligned}
$$

It can be verified that the operator $L_{2}$ also has the following two discrete eigen functions.

$$
\tilde{X}_{0}(\zeta)=(1-\zeta \tanh \zeta) \operatorname{sech} \zeta, \quad \tilde{X}_{1}(\zeta)=\operatorname{sech} \zeta \tanh \zeta
$$

It may be further noted that the eigen function $\tilde{X}_{1}$ corresponds to the discrete eigen value $\lambda=0$. That is

$$
L_{2} \tilde{X}_{1}(\zeta)=0
$$

\subsection{Complete set of orthonormal basis}

Having found the eigen functions $X(\zeta, k)$ and $\tilde{X}(\zeta, k)$, we now check the completeness of them by writing

$$
\int_{-\infty}^{\infty} X(\zeta, k) \tilde{X}^{*}\left(\zeta^{\prime}, k\right) d k+f\left(\zeta, \zeta^{\prime}\right)=\delta\left(\zeta-\zeta^{\prime}\right)
$$

where $f\left(\zeta, \zeta^{\prime}\right)$ is an arbitrary function to be determined. First we evaluate the integral in the left hand side of Eq. (48) after substituting the values of the eigen functions $X(\zeta, k)$ and $\tilde{X}(\zeta, k)$ given in Eqs. (45a) and (45b). Thus we have the following integrals to evaluate.

$$
\begin{aligned}
\int_{-\infty}^{\infty} X(\zeta, k) \tilde{X}^{*}\left(\zeta^{\prime}, k\right) d k= & \delta\left(\zeta-\zeta^{\prime}\right)-\frac{1}{\pi}\left[\int_{-\infty}^{\infty} \frac{d k}{\left(1+k^{2}\right)} e^{i k\left(\zeta-\zeta^{\prime}\right)}\right. \\
& \times\left\{2-\operatorname{sech}^{2} \zeta^{\prime}-i k\left(\tanh \zeta-\tanh \zeta^{\prime}\right)\right\} \\
& -2 \int_{-\infty}^{\infty} \frac{d k}{\left(1+k^{2}\right)^{2}} e^{i k\left(\zeta-\zeta^{\prime}\right)} \tanh \zeta^{\prime} \\
& \left.\times(1-i k \tanh \zeta)\left(i k+\tanh \zeta^{\prime}\right)\right]
\end{aligned}
$$


The integrals in the right hand side of Eq.(49) are evaluated with the aid of the residue theorem. It may be noted that the integrands in these two integrals as functions of the complex variable $k$ are analytic everywhere in the complex $k$-plane except at the two poles $k= \pm i$ of first and second order respectively. Let $R_{1}$ and $R_{2}$ be the residues corresponding to the functions $\frac{1}{\left(1+k^{2}\right)} e^{i k\left(\zeta-\zeta^{\prime}\right)}\left\{2-i k\left(\tanh \zeta-\tanh \zeta^{\prime}\right)-\operatorname{sech}^{2} \zeta^{\prime}\right\}$ and $\frac{1}{\left(1+k^{2}\right)^{2}} e^{i k\left(\zeta-\zeta^{\prime}\right)}(1-$ $i k \tanh \zeta)\left(i k+\tanh \zeta^{\prime}\right) \tanh \zeta^{\prime}$ in Eq. (49) at the pole $k=i$ of first and second order respectively. On calculating the residues $R_{1}$ and $R_{2}$ using standard procedure we obtain

$$
\begin{aligned}
R_{1}= & \frac{-i}{2}\left[2+\tanh \zeta-\tanh \zeta^{\prime}-\operatorname{sech}^{2} \zeta\right] e^{\left(\zeta-\zeta^{\prime}\right)} \\
R_{2}= & \frac{-i}{4}\left[\left(1-\zeta+\zeta^{\prime}\right)\left(\tanh \zeta-\tanh \zeta^{\prime}\right)-\left(\zeta-\zeta^{\prime}\right)\right. \\
& \left.\times\left(1-\tanh \zeta \tanh \zeta^{\prime}\right)\right] e^{\left(\zeta-\zeta^{\prime}\right)}
\end{aligned}
$$

On summing up the above results Eq. (49) becomes

$$
\begin{aligned}
\int_{-\infty}^{\infty} X(\zeta, k) \tilde{X}^{*}\left(\zeta^{\prime}, k\right) d k= & \delta\left(\zeta-\zeta^{\prime}\right)-\left[\operatorname{sech} \zeta \operatorname{sech} \zeta^{\prime}\left(1-\zeta^{\prime} \tanh \zeta^{\prime}\right)\right. \\
& \left.+\zeta \operatorname{sech} \zeta \operatorname{sech} \zeta^{\prime} \tanh \zeta^{\prime}\right]
\end{aligned}
$$

which can be identified as

$$
\int_{-\infty}^{\infty} X(\zeta, k) \tilde{X}^{*}\left(\zeta^{\prime}, k\right) d k=\delta\left(\zeta-\zeta^{\prime}\right)-\left[X_{0}(\zeta) \tilde{X}_{0}\left(\zeta^{\prime}\right)+X_{1}(\zeta) \tilde{X}_{1}\left(\zeta^{\prime}\right)\right]
$$

upon using the values of $\tilde{X}_{0}(\zeta)$ and $\tilde{X}_{1}(\zeta)$ as given in Eq. $(46)$. By substituting Eq.(52) in Eq.(48), we can evaluate the following value of $f\left(\zeta, \zeta^{\prime}\right)$ in terms of the discrete orthogonal states.

$$
f\left(\zeta, \zeta^{\prime}\right)=\sum_{j=0,1} X_{j}(\zeta) \tilde{X}_{j}(\zeta)
$$

Thus, by comparing Eqs.(51) and (52), we can write two additional orthogonal discrete states given by

$$
X_{0}(\zeta)=\operatorname{sech} \zeta, \quad X_{1}(\zeta)=\zeta \operatorname{sech} \zeta
$$

It may be checked that the following relations exist between the discrete states $X_{0}(\zeta), \tilde{X}_{0}(\zeta), X_{1}(\zeta)$ and $\tilde{X}_{1}(\zeta)$.

$$
L_{1} X_{0}=0, L_{1} X_{1}(\zeta)=-2 \tilde{X}_{1}(\zeta), L_{2} \tilde{X}_{0}(\zeta)=2 X_{0}(\zeta)
$$


in addition to the relation given in Eq.(47). In conclusion, we have a set of two complete orthonormal bases $\{X\}$ and $\{\tilde{X}\}$ given by $\left\{X(\zeta, k), X_{0}(\zeta), X_{1}(\zeta)\right\}$ and $\left\{\tilde{X}(\zeta, k), \tilde{X}_{0}(\zeta), \tilde{X}_{1}(\zeta)\right\}$ respectively. These set of orthonormal basis functions will be used to construct the perturbed soliton solution.

\subsection{Evaluation of $T(\tau)$}

Having solved Eq.(28a) by finding $X(\zeta)$, in order to construct $\psi^{(1)}(\zeta, \tau)$, we now find $T(\tau)$ by solving Eq. (28b). For this first we rewrite Eq. (28b) by replacing the function $H(\tau)$ in the right hand side using Eq. (26) and (41).

$$
T_{\tau}-\lambda_{0} T=\frac{F^{(1)}(\zeta, \tau)}{i k \tilde{X}(\zeta, k)}
$$

On multiplying and dividing the right hand side of Eq. (56) by $X^{*}(\zeta, k)$ and on integrating with respect to $\zeta$ between the limits $-\infty$ to $+\infty$ we obtain due to orthonormality of the functions $X$ and $\tilde{X}$ (see Eq.(43)) the following equation.

$$
T_{\tau}-\lambda_{0} T=\frac{1}{i k} \int_{-\infty}^{\infty} F^{(1)}(\zeta, \tau) X^{*}(\zeta, k) d \zeta
$$

which can be explicitly written after substituting the values of $F^{(1)}(\zeta, \tau)$ and $X^{*}(\zeta, k)$ from Eqs. (24b) and (45a) respectively as

$$
\begin{aligned}
T_{\tau}-\lambda_{0} T= & \frac{2}{i \sqrt{2 \pi} k\left(1+k^{2}\right)} \int_{-\infty}^{\infty} d \zeta\left([g(\zeta) \operatorname{sech} \zeta]_{\zeta}+2 v_{0} \operatorname{sech} \zeta\left[m_{t_{1}}\right.\right. \\
& \left.\left.+\left(m^{2} \xi_{t_{1}}-\zeta m_{t_{1}}\right) \tanh \zeta\right]\right)\left(1-k^{2}+2 i k \tanh \zeta\right) e^{-i k \zeta}
\end{aligned}
$$

On solving the above equation using standard procedure, we obtain

$$
\begin{aligned}
T(\tau, k)= & C(k) e^{\lambda_{0} \tau}-\frac{2}{i \sqrt{2 \pi} \lambda_{0} k\left(1+k^{2}\right)} \int_{-\infty}^{\infty} d \zeta\left([g(\zeta) \operatorname{sech} \zeta]_{\zeta}+2 v_{0} \operatorname{sech} \zeta\right. \\
& \left.\times\left[m_{t_{1}}+\left(m^{2} \xi_{t_{1}}-\zeta m_{t_{1}}\right) \tanh \zeta\right]\right)\left(1-k^{2}+2 i k \tanh \zeta\right) e^{-i k \zeta},
\end{aligned}
$$

where $\mathrm{C}(\mathrm{k})$ is a constant which can be found by using the initial condition $T(\tau, k)=0$ when $\tau \rightarrow-\frac{(1+v) \zeta}{2}$. Thus we obtain 


$$
\begin{aligned}
C(k)= & \frac{2}{i \sqrt{2 \pi} \lambda_{0} k\left(1+k^{2}\right)} \int_{-\infty}^{\infty} d \zeta\left([g(\zeta) \operatorname{sech} \zeta]_{\zeta}+2 v_{0} \operatorname{sech} \zeta\left[m_{t_{1}}\right.\right. \\
& \left.\left.+\left(m^{2} \xi_{t_{1}}-\zeta m_{t_{1}}\right) \tanh \zeta\right]\right)\left(1-k^{2}+2 i k \tanh \zeta\right) e^{\lambda_{0}\left(1+v_{0}\right) \frac{\zeta}{2}-i k \zeta}
\end{aligned}
$$

and hence

$$
\begin{aligned}
T(\tau, k)= & \frac{2}{\sqrt{2 \pi} i \lambda_{0} k\left(1+k^{2}\right)} \int_{-\infty}^{\infty} d \zeta^{\prime}\left(\left[g\left(\zeta^{\prime}\right) \operatorname{sech} \zeta^{\prime}\right]_{\zeta^{\prime}}+2 v_{0} \operatorname{sech} \zeta^{\prime}\left[m_{t_{1}}\right.\right. \\
& \left.\left.+\left(m^{2} \xi_{t_{1}}-\zeta^{\prime} m_{t_{1}}\right) \tanh \zeta^{\prime}\right]\right)\left(1-k^{2}+2 i k \tanh \zeta^{\prime}\right) e^{-i k \zeta^{\prime}} \\
& \times\left(e^{\lambda_{0}\left[\tau+\frac{\left(1+v_{0}\right)}{2} \zeta^{\prime}\right]}-1\right) .
\end{aligned}
$$

The allowed discrete values of $T$ will be determined in the next section while constructing the perturbed part of the soliton $\psi^{(1)}(\zeta, \tau)$ using the values of $X(\zeta)$ and $T(\tau)$.

\subsection{Perturbation of soliton}

As mentioned, we now write down the first order perturbation correction $\Psi^{(1)}(\zeta, \tau)$ in terms of the basis functions $\{X\} \equiv\left\{X(\zeta, k), X_{0}(\zeta), X_{1}(\zeta)\right\}$ and $\{T\} \equiv\left\{T(\tau, k), T_{0}(\tau), T_{1}(\tau)\right\}$. As per Eq.(26), in terms of the basis functions the perturbed part of the soliton can be written as

$$
\Psi^{(1)}(\zeta, \tau)=\int_{-\infty}^{\infty} X(\zeta, k) T(\tau, k) d k+\sum_{j=0,1} X_{j}(\zeta) T_{j}(\tau)
$$

However, it should be noted that in the basis $\{T\}, T_{0}(\tau)$ and $T_{1}(\tau)$ are yet to be

determined. Therefore before evaluating the values of $\Psi^{(1)}(\zeta, k)$, we determine $T_{0}(\tau)$ and $T_{1}(\tau)$. This is carried out by substituting Eq.(62) in Eq.(25) which finally becomes

$$
\begin{array}{r}
\int_{-\infty}^{\infty} i k\left[T_{\tau}(\tau, k)-\lambda_{0} T(\tau, k)\right] \tilde{X}(\zeta, k) d k+T_{1 \tau}(\tau) \tilde{X}_{0}(\zeta) \\
-\left[T_{0 \tau}(\tau)-2 T_{1}(\tau)\right] \tilde{X}_{1}(\zeta)=F^{(1)}(\zeta, \tau),
\end{array}
$$

upon using Eqs.(29),(41),(47) and (55). Now multiplying Eq.(63) by $X^{*}(\zeta, k), X_{0}(\zeta)$ and $X_{1}(\zeta)$ separately and using the orthonormal relations such as $\int_{-\infty}^{\infty} \tilde{X}(\zeta, k) X^{*}(\zeta, k) d k=$ $1, \int_{-\infty}^{\infty} \tilde{X}(\zeta, k) X_{j}(\zeta) d \zeta \equiv \int_{-\infty}^{\infty} X(\zeta, k) \tilde{X}_{j}(\zeta) d \zeta=0, \int_{-\infty}^{\infty} X_{j}(\zeta) \tilde{X}_{l}(\zeta) d \zeta=\delta_{j l}, j, l=$ 
0,1 , we get

$$
\begin{aligned}
T_{\tau}(\tau, k)-\lambda_{0} T(\tau, k) & =\frac{1}{i k} \int_{-\infty}^{\infty} F^{(1)}(\zeta, \tau) X^{*}(\zeta, k) d \zeta \\
T_{1 \tau}(\tau) & =\int_{-\infty}^{\infty} F^{(1)}(\zeta, \tau) X_{0}(\zeta) d \zeta \\
T_{0 \tau}(\tau)-2 T_{1}(\tau) & =-\int_{-\infty}^{\infty} F^{(1)}(\zeta, \tau) X_{1}(\zeta) d \zeta
\end{aligned}
$$

As $F^{(1)}(\zeta, \tau)$ given in Eq. (24b) does not contain time ' $\tau$ ' explicitly, the right hand side of Eqs. (64a-c) also should be independent of time. Then it is easy to verify that for the values of $F^{(1)}(\zeta, \tau), X^{*}(\zeta, k), T(\tau, k)$ and $\lambda_{0}$ given respectively in Eqs. (24b), (45a), (61) and (42), Eq. (64a) satisfies identically. As the right hand sides of Eqs. (64b) and (64c) are also independent of time, they give rise to secularities and hence the nonsecular conditions can be written as

$$
\begin{gathered}
\int_{-\infty}^{\infty} F^{(1)}(\zeta, \tau) X_{0}(\zeta) d \zeta=0 \\
\int_{-\infty}^{\infty} F^{(1)}(\zeta, \tau) X_{1}(\zeta) d \zeta=0 .
\end{gathered}
$$

Using Eqs. (65a) and (65b) back in Eqs. (64b) and (64c), we obtain $T_{1}(\tau)=0$ and $T_{0}(\tau)=C_{1}$ which has to be determined. For this, we substitute $T_{1}(\tau)=$ 0 in Eq. (64c) and integrate with respect to $\tau$ to obtain

$$
\begin{aligned}
T_{0}(\tau) \equiv C_{1}= & (1+v) \int_{-\infty}^{\infty} d \zeta\left([g(\zeta) \operatorname{sech} \zeta]_{\zeta}+2 v_{0} \operatorname{sech} \zeta\right. \\
& \left.\times\left[m_{t_{1}}+\left(m^{2} \xi_{t_{1}}-\zeta m_{t_{1}}\right) \tanh \zeta\right]\right) \zeta^{2} \operatorname{sech} \zeta
\end{aligned}
$$

\subsection{Variation of soliton parameters}

We now estimate the nonsecularity conditions (65a) and (65b) by evaluating the integrals after substituting the values of $F^{(1)}(\zeta, \tau), X_{0}(\zeta)$ and $X_{1}(\zeta)$ respectively from Eqs.(24b) and (54). The results give

$$
\begin{aligned}
m_{t_{1}} & =-\frac{1}{2 v_{0}} \int_{-\infty}^{\infty}[g(\zeta) \operatorname{sech} \zeta]_{\zeta} \operatorname{sech} \zeta d \zeta \\
\xi_{t_{1}} & =-\frac{1}{2 m^{2} v_{0}} \int_{-\infty}^{\infty}[g(\zeta) \operatorname{sech} \zeta]_{\zeta} \zeta \operatorname{sech} \zeta d \zeta
\end{aligned}
$$

Eq. (67a) describes the time evolution of the inverse of the width of the soliton and Eq. (67b) gives the velocity of the soliton. $g(\zeta)$ that appears in the above 
nonsecularity relation is related to the inhomogeneity in stacking energy of DNA. In order to evaluate the integrals in Eqs. (67a) and (67b) explicitly we have to substitute specific value for $g(\zeta)$. We consider $g(\zeta)$ in the form of localized and periodic functions. A localized $g(\zeta)$ corresponds to the intercalation of a compound between neighbouring base pairs without disturbing the base pairs and their sequence in the DNA double helical chain. The periodic nature of $g(\zeta)$ represents a periodic repetition of similar base pairs along the helical chain. We consider the localized form of $g(\zeta)$ as (i) $g(\zeta)=\operatorname{sech} \zeta$ and the periodic form of $g(\zeta)$ as (ii) $g(\zeta)=\cos \zeta$. We substitute the above values of $g(\zeta)$ one by one in Eqs. (67a) and (67b) and evaluate the integrals in the right hand side to understand the time evolution of the width of the soliton and its velocity. At this point it is worth mentioning that Dandoloff and Saxena [35] realized that in the case of XY-spin chains the model of which identifies with our plane-base rotator model, the ansatz $g(\zeta)=\operatorname{sech} \zeta$ energetically favours the deformation of spin chains.

When we substitute $g(\zeta)=\operatorname{sech} \zeta$ in Eqs. (67a) and (67b) and on evaluating the integrals, we obtain

$$
m_{t_{1}}=0, \quad \xi_{t_{1}}=\frac{\pi}{6 m^{2} v_{0}}
$$

The above equations can be rewritten in terms of the original time variable $\hat{t}$ by using the transformation $\frac{\partial}{\partial \hat{t}}=\frac{\partial}{\partial t_{0}}+\epsilon \frac{\partial}{\partial t_{1}}$ or in otherwords $m_{\hat{t}}=m_{t_{0}}+\epsilon m_{t_{1}}$ and $\xi_{\hat{t}}=\xi_{t_{0}}+\epsilon \xi_{t_{1}}$. As $\mathrm{m}$ is independent of $t_{0}\left(m_{t_{0}}=0\right)$ and $\xi_{t_{0}}=v_{0}$, we can write

$$
m=m_{0}, \quad \xi_{\hat{t}} \equiv v=v_{0}+\frac{\epsilon \pi}{6 m_{0}^{2} v_{0}}
$$

where $1 / m_{0}$ is the initial width of the soliton. The first of Eq.(69) says that when $g(\zeta)=\operatorname{sech} \zeta$, the width $\left(\mathrm{m}^{-1}\right)$ of the soliton remains constant. However from the second of Eq.(69), we find that the velocity of the soliton gets a correction. As the correction term is a definite positive quantity the velocity of the soliton increases in this case. Interestingly, the amount of increment in velocity depends on the initial width and initial velocity of the soliton. Wider the soliton, greater the increment in velocity due to inhomogeneity. Also slowly moving solitons gain more speed. The increase in speed helps to overcome the barrier of the local inhomogeneity (which may be due to the presence of abasic site or intercalation of a molecule) and the solitons representing the open state will propagate easily along the chain without formation of a bound state. In a similar study on resonant kink-impurity interaction and kink scattering in the sine-Gordon model Zhang Fei et al. [32] observed that if the initial velocity of the kink is smaller than a critical velocity it will be either trapped or reflected 
by the impurity. In fact they have showed that for most of the initial velocities, the kink is trapped except in the case of some special initial velocities the kink may be totally reflected by the impurity. It was further found that when the kink velocity is greater than the critical velocity, it will pass through the impurity. It is interesting to note that our results on the velocity of soliton found in Eq. (69) is similar to the last case of Zhang Fei et al [32] where the soliton will pass through by overcoming the barrier of the local inhomogeneity.

Next, we substitute the periodic function $g(\zeta)=\cos \zeta$ in Eqs.(67a) and (67b) and evaluate the integrals to obtain

$$
m_{t_{1}}=0, \quad \xi_{t_{1}}=\frac{\pi^{2}}{16 m^{2} v_{0}}
$$

From the above the parameters $m$ and $\xi$ can be written in terms of the original variable $\hat{t}$ after solving Eq.(70) as

$$
m=m_{0}, \quad \xi_{\hat{t}} \equiv v=v_{0}+\frac{\epsilon \pi^{2}}{16 m^{2} v_{0}} .
$$

From Eq. (71) we find that the width of the soliton in this case remains constant and the soliton velocity increases similar to the case $g(\zeta)=\operatorname{sech} \zeta$. On comparing Eqs. (69) and (71), we observe that the increase in velocity of the soliton is less in this case. This is because in this case, the inhomogeneity occurs periodically in the entire DNA chain in terms of sequence.

\subsection{First order perturbed soliton}

Now, we explicitly construct the first order perturbation correction to the one soliton for the different cases of $g(\zeta)$ by substituting the values of the basis functions $\{X\} \equiv\left\{X(\zeta, k), X_{0}(\zeta), X_{1}(\zeta)\right\}$ and $\{T\} \equiv\left\{T(\tau, k), T_{0}(\tau), T_{1}(\tau)\right\}$ after using the values of $F^{(1)}(\zeta, \tau), m_{t_{1}}$ and $\xi_{t_{1}}$ for the respective $g(\zeta)$ values in Eq. (62). Thus in the case of $g(\zeta)=\operatorname{sech} \zeta$, we substitute the values of $X(\zeta, k), X_{0}(\zeta)$ and $X_{1}(\zeta)$ from Eqs. (45a) and (54) and $T(\tau, k), T_{0}(\tau)$ from Eqs. (61) and (66) and $F^{(1)}(\zeta, \tau)$ from Eq. (24b) and use the values of $m_{t_{1}}$ and $\xi_{t_{1}}$ from Eqs. (68) in Eq. (62) to obtain 


$$
\begin{aligned}
\Psi^{(1)}\left(\zeta, t_{0}\right)= & -\frac{1}{3 \pi} \int_{-\infty}^{\infty} \frac{d k}{\left(1+k^{2}\right)^{3}}\left(1-k^{2}-2 i k \tanh \zeta\right) e^{i k \zeta} \int_{-\infty}^{\infty} d \zeta \\
& \times\left(1-k^{2}+2 i k \tanh \zeta\right)(\pi-6 \operatorname{sech} \zeta) \operatorname{sech} \zeta \tanh \zeta \\
& \times\left[e^{-i k \zeta}-e^{i \frac{\left(1+k^{2}\right)}{k} \alpha} e^{i \beta \zeta}\right],
\end{aligned}
$$

where $\alpha=\frac{t_{0}-m\left(1+v_{0}\right) \zeta}{2 m}$ and $\beta=\frac{\left(1+v_{0}\right)\left(1+k^{2}\right)}{2 k}-k$. While writing the above, we have also used $T_{1}(\tau)=0, \tau=\frac{1}{2 m}\left[t_{0}-m\left(1+v_{0}\right) \zeta\right]$ and the eigen value $\lambda_{0}=\frac{i\left(1+k^{2}\right)}{k}$. The integrals in the right hand side of Eq.(72) can be evaluated using standard residue theorem . The details are given in Appendix-A. The final form of $\Psi^{(1)}\left(\zeta, t_{0}\right)$ after evaluating the integrals becomes

$$
\begin{aligned}
\Psi^{(1)}\left(\zeta, t_{0}\right) \approx & \frac{80}{27 \sqrt{2}} \sqrt{\operatorname{sech} \zeta} e^{-\frac{3 \zeta}{2}}+\frac{64}{27 \sqrt[3]{2}} \tanh \zeta \sqrt[3]{\operatorname{sech} \zeta} e^{-\frac{5}{3} \zeta} \\
& +\frac{\pi}{6 v^{2}}\left[2 v(1+v) \zeta+v^{2}+4 \alpha v-1\right] \operatorname{sech} \zeta
\end{aligned}
$$

While constructing the above perturbed solution, we have used the values of m's and $\xi$ 's and of course the corresponding $F^{(1)}\left(\zeta, t_{0}\right)$ values. Finally, the perturbed one soliton solution that is $\Psi\left(z, t_{0}\right)=\Psi^{(0)}\left(z, t_{0}\right)+\Psi^{(1)}\left(z, t_{0}\right)$ (choosing $\epsilon=1$ ) is written using Eqs. (22) and (73) as

$$
\begin{aligned}
\Psi\left(z, t_{0}\right) & \approx 4 \arctan \exp \left[ \pm m_{0}\left(z-v_{0} t_{0}\right)\right]+\frac{80}{27 \sqrt{2}} \sqrt{\operatorname{sech}\left[ \pm m\left(z-v t_{0}\right)\right]} \\
& \times e^{\mp \frac{3\left(m\left(z-v t_{0}\right)\right.}{2}}+\frac{64}{27 \sqrt[3]{2}} \tanh \left[ \pm m\left(z-v t_{0}\right)\right] \sqrt[3]{\operatorname{sech}\left[ \pm m\left(z-v t_{0}\right)\right]} \\
& \times e^{\mp \frac{5}{3} m\left(z-v t_{0}\right)}+\frac{\pi}{6 m v^{2}}\left[m\left(v^{2}-1\right)+2 t_{0} v\right] \operatorname{sech}\left[ \pm m\left(z-v t_{0}\right)\right] .
\end{aligned}
$$

In Eq.(74) while the upper sign corresponds to perturbed kink-soliton the lower sign represents the perturbed antikink-soliton. The rotation of bases denoted by $\phi\left(z, t_{0}\right)$ can be immediately written down by using the relation $\phi=\frac{\Psi}{2}$. In Figs. $4 \mathrm{a}$ and $4 \mathrm{~b}$, we plot $\phi\left(z, t_{0}\right)$ (rotation of bases under perturbation) for the parametric choice $v_{0}=0.4$. From the figure, we observe that there appears fluctuation in the form of a train of pulses closely resembling the shape of the inhomogeneity profile in the width of the soliton as time progresses. Also, as time passes, the amplitude of the pulses generating this fluctuation increases. However, in the asymptotic region of the soliton there is no change in the topological character and no fluctuations appear in that region. It shows that the localized inhomogeneity in stacking energy in DNA in the form of a pulse $(g(z)=\operatorname{sech} z)$ does not affect very much the opening of bases except fluctuations in the form of a train of pulses in the localized region of the kink and antikink-soliton. We have schematically represented this in Fig.4c. 


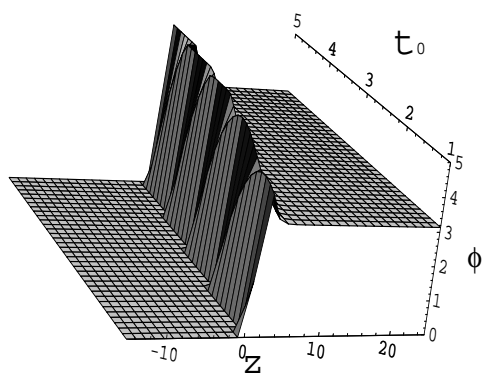

(a)

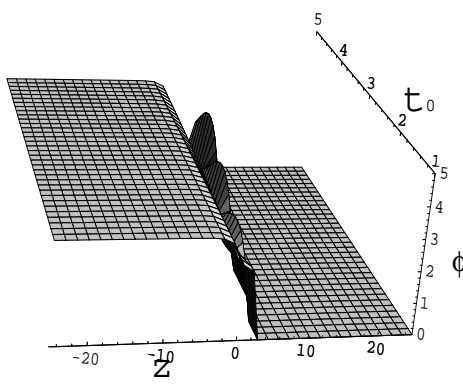

(b)

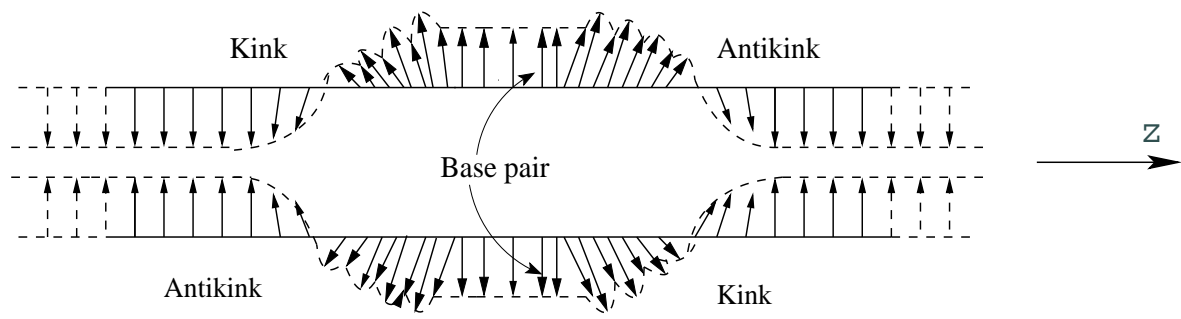

(c)

Fig. 4. (a) The perturbed kink-soliton and (b) the perturbed antikink-soliton for the inhomogeneity $g(z)=\operatorname{sech} z$ and $v_{0}=0.4$. (c) A sketch of the base pair opening in DNA double helix with fluctuation.

We then repeat the procedure for constructing the perturbed one-soliton solution in the case of $g(\zeta)=\cos \zeta$ which is written as

$$
\begin{aligned}
\Psi^{(1)}\left(\zeta, t_{0}\right) \approx & \frac{1}{\pi} \int_{-\infty}^{\infty} \frac{d k}{\left(1+k^{2}\right)^{3}}\left(1-k^{2}-2 i k \tanh \zeta\right) e^{i k \zeta} \int_{-\infty}^{\infty} d \zeta\left(1-k^{2}\right. \\
& +2 i k \tanh \zeta)\left[\sin \zeta+\left(\cos \zeta-\frac{\pi^{2}}{8}\right) \tanh \zeta\right] \operatorname{sech} \zeta \\
& \times\left\{e^{i \frac{\left(1+k^{2}\right)}{k} \alpha} e^{i \beta \zeta}-e^{-i k \zeta}\right\}+(1+v) \operatorname{sech} \zeta \\
& \times \int_{-\infty}^{\infty} d \zeta \zeta^{2}\left[\sin \zeta+\left(\cos \zeta-\frac{\pi^{2}}{8}\right) \tanh \zeta\right] \operatorname{sech}^{2} \zeta
\end{aligned}
$$

The details of values of the integrals in the above equation are given in Appendix-B. The perturbed kink (upper sign)-antikink (lower sign) one soliton solution in this case is finally obtained as

$$
\begin{aligned}
\Psi\left(z, t_{0}\right) \approx & 4 \arctan \exp \left[ \pm m_{0}\left(z-v_{0} t_{0}\right)\right]+\frac{\pi^{2}}{16 m v^{2}}\left[m\left(v^{2}-1\right)+2 v t_{0}\right] \\
& \times \operatorname{sech}\left[m\left(z-v t_{0}\right)\right] .
\end{aligned}
$$

After finding $\phi\left(z, t_{0}\right)$ from the relation $\phi=\frac{\Psi}{2}$ we plot it in Figs.5a and 5b for 


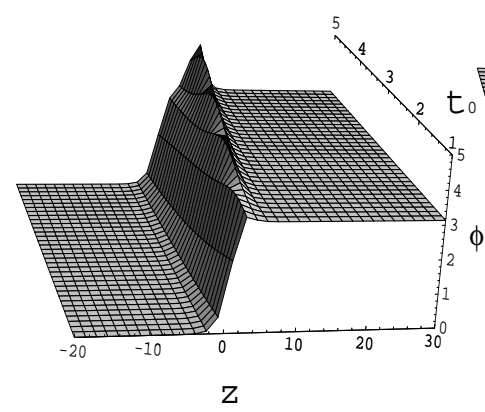

(a)

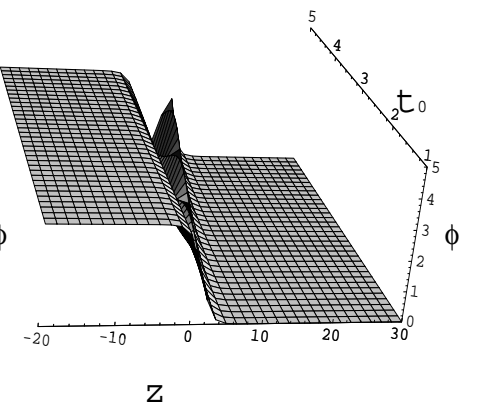

(b)

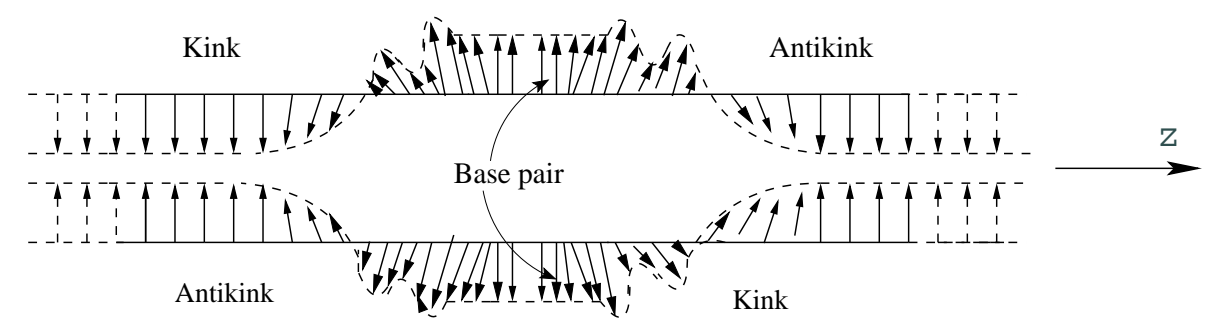

(c)

Fig. 5. The perturbed (a) kink-soliton and (b) antikink-soliton for the inhomogeneity $g(z)=\cos z$ with $v_{0}=0.4$. (c) A sketch of the open state configuration in DNA with small fluctuations.

the same value of the parameter as before. From the figures we observe that periodic oscillations appear in the width of the soliton without any change asymptotically.

\section{Conclusions}

In this paper, we studied the nonlinear dynamics of DNA double helix with stacking inhomogeneity by considering the dynamic plane-base rotator model. The dynamical equation which finally appeared in the form of a perturbed sine-Gordon equation was derived from a suitable Hamiltonian in analogy with Heisenberg model of an inhomogeneous anisotropic coupled spin chain or spin ladder with ferromagnetic legs and antiferromagnetic rung coupling in the continuum limit. In the unperturbed limit which is also the homogeneous limit, the dynamics is governed by the kink-antikink soliton of the integrable sine-Gordon equation which represents the open state configuration of base pairs in DNA double helix. Even though DNA double helix is a large molecular chain involving very large number of base pairs, base pair opening is limited to a very few number of base pairs forming localized coherent structure in the form of kink-antikink solitons which is obtained as a balance between disper- 
sion and nonlinearity traveling with constant speed and amplitude without loosing its energy along the helical chain. To understand the effect of stacking energy inhomogeneity on the open state configuration of base pairs we carried out a multiple scale soliton perturbation analysis. For implementing this we linearized the perturbed sine-Gordon equation using multiple-scale expansion to obtain linearized equations in the form of eigen value problems. The perturbed kink-antikink soliton solutions were constructed for different forms of inhomogeneities by solving the associated eigen value problem. By using the complete set of eigen functions thus obtained as the basis functions the perturbed solutions were constructed.

The perturbation not only modifies the shape of the soliton but also undergoes a slow time change of the soliton parameters namely the width and velocity for the different forms of the inhomogeneity chosen. We chose the inhomogeneity in the form of localized and periodic functions. The results show that when the inhomogeneity is either in the form $g(z)=\operatorname{sech} z$ or $g(z)=\cos z$, the width of the soliton remains constant. Thus, the number of base pairs participating in the opening do not change due to the above pattern of inhomogeneities. However, in this case the speed of the soliton increases with a correction that is proportional to the square of the initial width of the soliton and inversely proportional to its initial velocity. Thus the inhomogeneity increases the speed with which the base pairs are opening and closing or winding and unwinding. From the perturbed solutions corresponding to different inhomogeneities (see Figs. 4,5) we observe that the perturbation due to inhomogeneity in stacking energy along the strands introduces fluctuation only in the width of the solitons. The nature of the fluctuation varies depending on the type of inhomogeneity. In particular, when the inhomogeneity is in the form of $g(z)=$ sech $z$, we find that fluctuation in the form of pulse trains resembling the shape of the inhomogeneity is generated in the width of the soliton representing open state configuration. It is noted that in the long time limit, eventhough neighbouring pulses overlap the train of pulse-like fluctuations maintain their character without affecting the soliton. In a similar way, when the inhomogeneity is of the form $g(z)=\cos z$, the fluctuation appears in the form of periodic oscillations in the width of the soliton. In all these cases, asymptotically the kink-antikink soliton shape is preserved. The results indicate that inhomogeneity in stacking energy in DNA double helix can (i) introduce small fluctuations which may merge asymptotically during the process of opening and closing of base pairs (ii) increase or decrease the number of base pairs participating in the open state configuration and (iii) change the speed with which the open state configuration can travel along the double helical chain. Thus in conclusion the inhomogeneity in stacking does not affect the general pattern of base pair opening. Even though the size of the base pair is big and the solvent effect on pure rotation of base pairs is negligible, the soliton, a coherent structure which is formed by involving few 
base pairs move along the helical chain without dissipation or any other form of deformation. Similar conclusion was also arrived in the case of propagation of soliton representing base pair opening in a discrete site-dependent DNA [21] and propagation of bubble in a heterogeneous DNA chain [24]. As nature selects generally inhomogeneous DNA, the functions such as replication and transcription can be explained more viably through formation of open states through our inhomogeneous model rather than the homogeneous case. This is also because, it is known that transcription and replication are sequence dependent. This is further similar to what was observed in the case of proteins where inhomogeneity of the sequence leads to inhomogeneous fluctuations, enhanced by the nonlinear effect [49]. Eventhough the relevance of these effects in the DNA to biological processes are not yet clearly, established a recent study suggests that thermally induced base pair opening agrees with experimental observation on DNA base pair opening detected by potassium permanganate foot printing [50]. We will make numerical analysis of the discrete dynamical equation separately to understand the discreteness effect which will be published elsewhere. It is also equally important to analyse the nonlinear dynamics of DNA double helix when the hydrogen bonding energy depends on the distance between the bases (inhomogeneity in hydrogen bonds) and the study is under progress. Also, the nonlinear dynamics study of open state configuration facilitated by enzymes (protein) is under progress.

\section{Acknowledgements}

The authors thank the anonymous referees for useful comments. The major portion of the work is done within the framework of the Associateship Scheme of the Abdus Salam International Centre for Theoretical Physics, Trieste, Italy and the financial support is acknowledged. The work of M. D also forms part of a major DST project. V. V also thanks SBI for financial support.

\section{A Evaluation of integrals in Eq.(72) using residue theorem}

In this appendix we evaluate the integrals found in Eq.(72) using standard residue theorem. Eq.(72) is of the form

$$
\begin{aligned}
\Psi^{(1)}\left(\zeta, t_{0}\right)= & -\frac{1}{3 \pi} \int_{-\infty}^{\infty} \frac{d k}{\left(1+k^{2}\right)^{3}}\left(1-k^{2}-2 i k \tanh \zeta\right) e^{i k \zeta} \int_{-\infty}^{\infty} d \zeta\left(1-k^{2}\right. \\
& +2 i k \tanh \zeta)(\pi-6 \operatorname{sech} \zeta) \operatorname{sech} \zeta \tanh \zeta \\
& \times\left[e^{-i k \zeta}-e^{i \frac{\left(1+k^{2}\right)}{k} \alpha} e^{i \beta \zeta}\right]
\end{aligned}
$$


The evaluation of various integrals in Eq. (A.1) can be facilitated by first finding the values of the following two integrals [51].

$$
\begin{aligned}
& I_{1}=\int_{-\infty}^{\infty} \operatorname{sech} \zeta e^{i \chi \zeta} d \zeta, \\
& I_{2}=\int_{-\infty}^{\infty} \tanh \zeta e^{i \chi \zeta} d \zeta,
\end{aligned}
$$

where $\chi$ can take values $-k$ and $\beta$. The integrand in $I_{1}$ is found to be analytic everywhere except at the pole $\zeta \rightarrow i(2 n+1) \frac{\pi}{2}, n=0,1,2, \ldots$ The residue of the function $\operatorname{sech} \zeta e^{i \chi \zeta}$ can be written as

$$
\operatorname{Res}\left(\zeta=i(2 n+1) \frac{\pi}{2}\right)=\sum_{n=0}^{\infty} \lim _{\zeta \rightarrow i(2 n+1) \frac{\pi}{2}} \frac{e^{i \chi \zeta}}{\frac{d}{d \zeta}(\cosh \zeta)}
$$

which can be simplified to give

$$
\operatorname{Res}\left(\zeta=i(2 n+1) \frac{\pi}{2}\right)=\frac{1}{2 i \cosh \frac{\pi}{2} \chi} .
$$

Thus the integral value of $I_{1}$ in Eq. (A.2) is written as

$$
I_{1} \equiv \int_{-\infty}^{\infty} \operatorname{sech} \zeta e^{i \chi \zeta} d \zeta=\frac{\pi}{\cosh \left(\frac{\pi}{2} \chi\right)}
$$

Similarly, using the same procedure we evaluate the integral $I_{2}$ in which the integrand $\tanh \zeta e^{i \chi \zeta}$ contains poles at $\zeta \rightarrow i(2 n+1) \frac{\pi}{2}, n=0,1,2, \ldots$ and obtain

$$
I_{2} \equiv \int_{-\infty}^{\infty} \tanh \zeta e^{i \chi \zeta} d \zeta=\frac{i \pi}{\sinh \left(\frac{\pi}{2} \chi\right)}
$$

Now using the value of the integral $I_{1}$ as given in Eq. (A.6), we evaluate the following integrals found in Eq. (A.1) by integrating them by parts successively to obtain

$$
\begin{gathered}
\int_{-\infty}^{\infty} \operatorname{sech} \zeta \tanh \zeta e^{i \chi \zeta} d \zeta=\frac{i \pi \chi}{\cosh \left(\frac{\pi}{2} \chi\right)} \\
\int_{-\infty}^{\infty} \operatorname{sech} \zeta \tanh ^{2} \zeta e^{i \chi \zeta} d \zeta=\frac{\pi\left(1-\chi^{2}\right)}{2 \cosh \left(\frac{\pi}{2} \chi\right)}
\end{gathered}
$$


Similarly, using Eq. (A.7) we evaluate the following integrals found in Eq. (A.1) by making successive integrations by parts and obtain

$$
\begin{aligned}
\int_{-\infty}^{\infty} \operatorname{sech}^{2} \zeta \tanh \zeta e^{i \chi \zeta} d \zeta & =\frac{i \pi \chi^{2}}{2 \sinh \left(\frac{\pi}{2} \chi\right)} \\
\int_{-\infty}^{\infty} \operatorname{sech}^{2} \zeta \tanh ^{2} \zeta e^{i \chi \zeta} d \zeta & =\frac{\pi \chi\left(2-\chi^{2}\right)}{6 \sinh \left(\frac{\pi}{2} \chi\right)}
\end{aligned}
$$

Here also $\chi$ can take values $-k$ and $\beta$. Substituting the values of the integrals found in Eqs. (A.8a), (A.8b) (A.9a) and (A.9b) in Eq. (A.1), we obtain

$$
\begin{aligned}
\Psi^{(1)}\left(\zeta, t_{0}\right)= & -\frac{i}{12}\left[\int _ { - \infty } ^ { \infty } d k \frac { ( 1 - k ^ { 2 } - 2 i k \operatorname { t a n h } \zeta ) } { k ^ { 2 } ( 1 + k ^ { 2 } ) ^ { 2 } } \left(\pi\left(1-v^{2}\right) k\left(1+k^{2}\right)\right.\right. \\
& \times \operatorname{sech} \frac{\pi}{2} \beta-\left\{(2-v)(1+v)^{2}\left(1+k^{2}\right)^{2}-4 k^{2}\left(1+v+k^{2}\right)\right\} \\
& \left.\times \operatorname{cosech} \frac{\pi}{2} \beta\right) e^{i \frac{\left(1+k^{2}\right)}{k} \alpha+i k \zeta} \\
& \left.+4 \int_{-\infty}^{\infty} d k \frac{\left(k^{2}-k^{4}-2 i k^{3} \tanh \zeta\right)}{\left(1+k^{2}\right)^{2} \sinh \frac{\pi}{2} k} e^{i k \zeta}\right]
\end{aligned}
$$

Now, before writing down the final form of $\psi^{(1)}\left(\zeta, t_{0}\right)$ we evaluate the integrals with respect to $k$ in the right hand side of the above equation. For this first we rearrange the integrand in Eq.(A.10) by simple multiplication and call them $I_{3}, I_{4}, I_{5}$ and $I_{6}$ as given below.

$$
\begin{aligned}
& I_{3}=\int_{-\infty}^{\infty} d k \frac{\left(k^{2}-k^{4}-2 i k^{3} \tanh \zeta\right)}{\left(1+k^{2}\right)^{2} \sinh \frac{\pi}{2} k} e^{i k \zeta} \\
& I_{4}=\int_{-\infty}^{\infty} d k \frac{\left(1-k^{2}-2 i k \tanh \zeta\right)}{\left(1+k^{2}\right)^{2} \sinh \frac{\pi}{2} \beta}\left(1+v+k^{2}\right) e^{i \frac{\left(1+k^{2}\right)}{k} \alpha+i k \zeta} \\
& I_{5}=\int_{-\infty}^{\infty} d k \frac{\left(1-k^{2}-2 i k \tanh \zeta\right)}{k^{2} \sinh \frac{\pi}{2} \beta} e^{i \frac{\left(1+k^{2}\right)}{k} \alpha+i k \zeta} \\
& I_{6}=\int_{-\infty}^{\infty} d k \frac{\left(1-k^{2}-2 i k \tanh \zeta\right)}{k\left(1+k^{2}\right) \cosh \frac{\pi}{2} \beta} e^{i \frac{\left(1+k^{2}\right)}{k} \alpha+i k \zeta}
\end{aligned}
$$

The integral $I_{3}$ can be evaluated by finding the residue of the integrand $\frac{\left(k^{2}-k^{4}-2 i k^{3} \tanh \zeta\right)}{\left(1+k^{2}\right)^{2} \sinh k \frac{\pi}{2}} e^{i k \zeta}$ at the poles $k=i$ of order two and at the simple pole $k=2 i n$. The results are given by 


$$
\begin{aligned}
& \operatorname{Res}(k=i)=\frac{1}{2}(\zeta-2) \operatorname{sech} \zeta \\
& \operatorname{Res}(k=2 i n)=\frac{40}{9 \sqrt{2} \pi} \sqrt{\operatorname{sech} \zeta} e^{-\frac{3 \zeta}{2}}+\frac{32}{9 \sqrt[3]{2} \pi} \tanh \zeta \sqrt[3]{\operatorname{sech} \zeta} e^{-\frac{5 \zeta}{3}}
\end{aligned}
$$

While writing the first term in Eq.(A.12b), we have approximated the results obtained as a series in terms of suitable functions. Adding the above two residue values we obtain the value of the integral $I_{3}$ as

$$
\begin{aligned}
I_{3}= & 2 \pi i\left[\frac{1}{2}(\zeta-2) \operatorname{sech} \zeta+\frac{40}{9 \pi \sqrt{2}} \sqrt{\operatorname{sech} \zeta} e^{-\frac{3 \zeta}{2}}+\frac{32}{9 \sqrt[3]{2} \pi} \tanh \zeta\right. \\
& \left.\times \sqrt[3]{\operatorname{sech} \zeta} e^{-\frac{5 \zeta}{3}}\right]
\end{aligned}
$$

In the case of the integral $I_{4}$, the integrand possesses a second order pole at $k=i$ and there is one more simple pole at $k=\frac{(2 n+1)}{(1-v)}\left(-i \pm \sqrt{\frac{1-v^{2}}{(2 n+1)^{2}}-1}\right)$ which is however out of the contour. Thus the residue for the integrand function $\frac{\left(1-k^{2}-2 i k \tanh \zeta\right)}{\left(1+k^{2}\right)^{2} \sinh \beta \frac{\pi}{2}}\left(1+v+k^{2}\right) e^{i \frac{\left(1+k^{2}\right)}{k} \alpha+i k \zeta}$ is obtained as

$$
\operatorname{Res}(k=i)=\frac{1}{2}(2+v \zeta+2 v \alpha) \operatorname{sech} \zeta
$$

and hence the right hand side of Eq. (A.14) represents the value of the integral $I_{4}$.

The integrand of the integral $I_{5}$ namely $\frac{\left(1-k^{2}-2 i k \tanh \zeta\right)}{k^{2} \sinh \beta \frac{\pi}{2}} e^{i \frac{\left(1+k^{2}\right)}{k} \alpha+i k \zeta}$ admits an essential singularity at $k=0$, in addition to a simple pole at $k=\frac{(2 n+1)}{(1-v)}(-i \pm$ $\left.\sqrt{\frac{1-v^{2}}{(2 n+1)^{2}}-1}\right)$ which is also out of the contour. The residue corresponding to the essential singularity is found by collecting the coefficient of $\frac{1}{k}$ after expanding the above function. Thus we obtain 


$$
\begin{aligned}
\operatorname{Res}(k=0)= & \sum_{n, n^{\prime}=0}^{\infty} \frac{(-1)^{n+n^{\prime}}\left(\frac{\pi}{2}\right)^{2 n-1} b^{n-1}(b-1)^{n} \alpha^{n^{\prime}}}{\left(n^{\prime} !\right)}(\zeta+\alpha)^{n^{\prime}}\left[\frac{B_{n}}{(n !)^{2}\left(n^{\prime} !\right)}\right. \\
& \times\left(2^{2 n-1}-1\right)+\frac{\left(\frac{\pi}{2}\right)^{2} b^{2} B_{n+1}}{(n+1) !^{2}\left(n^{\prime} !\right)}\left(2^{2 n+1}-1\right)[1-2 \tanh \zeta \\
& \left.\times \frac{(\zeta+\alpha)}{\left(n^{\prime}+1\right)}+\frac{(\zeta+\alpha)^{2}}{\left(n^{\prime}+1\right)\left(n^{\prime}+2\right)}\right]+\frac{\left(\frac{\pi}{2}\right)^{4} b^{4} B_{n+2}}{(n+2) !(n+3) !} \\
& \times \frac{(n+1)\left(2^{2 n+3}-1\right)}{\left(n^{\prime}+2\right) !}\left[1-2 \tanh \zeta \frac{(\zeta+\alpha)}{\left(n^{\prime}+3\right)}\right. \\
& \left.\left.+\frac{(\zeta+\alpha)^{2}}{\left(n^{\prime}+3\right)\left(n^{\prime}+4\right)}\right]+\ldots\right]
\end{aligned}
$$

where $B_{n}^{\prime}$ s are Bernoulli numbers and $b=\frac{(1+v)}{2}$. Thus the right hand side of Eq. (A.15) gives the value of the integral $I_{5}$. In the case of the integral $I_{6}$, the integrand $\frac{\left(1-k^{2}-2 i k \tanh \zeta\right)}{k\left(1+k^{2}\right) \cosh \frac{\pi}{2} \beta} e^{i \frac{\left(1+k^{2}\right)}{k} \alpha+i k \zeta}$ possesses a second order pole at $k=i$ and an essential singularity at $k=0$. In addition, we have a simple pole at $k=\frac{(2 n+1)}{(1-v)}\left(-i \pm \sqrt{\frac{1-v^{2}}{(2 n+1)^{2}}-1}\right)$ which is again out of the contour. The residues at the pole $k=i$ and at the essential singularity $k=0$ are respectively found to be

$$
\begin{aligned}
\operatorname{Res}(k=i)= & \frac{-1}{\pi v^{2}}(1-2 v \zeta-4 v \alpha) \operatorname{sech} \zeta, \\
\operatorname{Res}(k=0)= & \sum_{m, n, n^{\prime}=0 j=0}^{\infty} \sum_{j=0}^{m} \frac{(-1)^{n+n^{\prime}}(b-1)^{n} E_{n+m}\left(\frac{\pi}{2}\right)^{2 n+2 m} b^{n+2 m} \alpha^{n^{\prime}}}{(n !)(n+2 m) !\left(n^{\prime}\right) !\left(n^{\prime}+2 j\right) !} \\
& \times(\zeta+\alpha)^{n^{\prime}+2 j}\left[1-\frac{\frac{\pi}{2} b^{2}}{(n+1+2 m)(n+2+2 m)}\right. \\
& \left.-\frac{2\left(\frac{\pi}{2}\right)^{2} b^{2}(\zeta+\alpha) \tanh \zeta}{(n+1+2 m)(n+2+2 m)\left(n^{\prime}+1+2 j\right)}\right],
\end{aligned}
$$

where $E_{n}^{\prime} \mathrm{s}$ are Euler numbers. We evaluated the value of the residue given in (A.16a) using Mathematica. The value of the integral $I_{6}$ is the sum of the right hand sides of Eqs. (A.16a) and (A.16b).

Now the value of $\psi^{(1)}\left(\zeta, t_{0}\right)$ is found by combining Eqs. (A.13), (A.14), (A.15), (A.16a) and (A.16b) and the final form is written as

$$
\begin{aligned}
\Psi^{(1)}\left(\zeta, t_{0}\right) \approx & \frac{80}{27 \sqrt{2}} \sqrt{\operatorname{sech} \zeta} e^{-\frac{3 \zeta}{2}}+\frac{64}{27 \sqrt[3]{2}} \tanh \zeta \sqrt[3]{\operatorname{sech} \zeta} e^{-\frac{5}{3} \zeta} \\
& +\frac{\pi}{6 v^{2}}\left[2 v(1+v) \zeta+v^{2}+4 \alpha v-1\right] \operatorname{sech} \zeta
\end{aligned}
$$

While writing the above, we have dropped few higher order terms due to 
smallness in values that appeared in the residue of the essential singularity.

\section{B Evaluation of the integrals in Eq. (75) using residue theorem}

In this appendix, we evaluate the integrals found in Eq. (75) using standard residue theorem as done in the previous two cases. Eq.(75) is written as

$$
\begin{aligned}
\Psi^{(1)}\left(\zeta, t_{0}\right) \approx & \frac{1}{\pi} \int_{-\infty}^{\infty} \frac{d k}{\left(1+k^{2}\right)^{3}}\left(1-k^{2}-2 i k \tanh \zeta\right) e^{i k \zeta} \int_{-\infty}^{\infty} d \zeta\left(1-k^{2}\right. \\
& +2 i k \tanh \zeta)\left[\sin \zeta+\left(\cos \zeta-\frac{\pi^{2}}{8}\right) \tanh \zeta\right] \operatorname{sech} \zeta \\
& \times\left\{e^{i \frac{\left(1+k^{2}\right)}{k} \alpha} e^{i \beta \zeta}-e^{-i k \zeta}\right\}+(1+v) \operatorname{sech} \zeta\left[\int_{-\infty}^{\infty} d \zeta \zeta^{2}\right. \\
& \left.\times[\sin \zeta+\cos \zeta] \operatorname{sech}^{2} \zeta-\frac{\pi^{2}}{8} \int_{-\infty}^{\infty} d \zeta \zeta^{2} \operatorname{sech}^{2} \zeta \tanh \zeta\right]
\end{aligned}
$$

It may be verified that the last integral $\int_{-\infty}^{\infty} d \zeta \zeta^{2} \operatorname{sech}^{2} \zeta \tanh \zeta$ in the right hand side of Eq.(B.1) on evaluation vanishes. For evaluating some of the integrals found in Eq. (B.1) we use the values of the integrals given in Eqs. (A.6), (A.8a) and (A.8b) and also the value of the following integral.

$$
I_{8}=\int_{-\infty}^{\infty} \zeta^{2} \tanh \zeta e^{i \zeta} d \zeta
$$

The integrand in Eq.(B.2) is found to be analytic everywhere except at the pole $\zeta=i(2 n+1) \frac{\pi}{2}, \mathrm{n}=0,1,2, \ldots$ The residue of the function $\zeta^{2} \tanh \zeta e^{i \zeta}$ is then found to be $-\frac{\pi^{2}}{8 \sinh \left(\frac{\pi}{2}\right)}\left[1+2 \frac{e^{-\frac{\pi}{2}}}{\sinh \left(\frac{\pi}{2}\right)}+\frac{e^{\frac{\pi}{2}}\left(1-e^{-2 \pi \chi}\right)}{2 \sinh ^{3}\left(\frac{\pi}{2}\right)}\right]$ and hence the value of the integral $I_{8}$ is written as

$$
\left.I_{8} \equiv \int_{-\infty}^{\infty} \zeta^{2} \tanh \zeta e^{i \zeta} d \zeta=-\frac{i \pi^{3}}{4 \sinh \left(\frac{\pi}{2}\right)}\left[1+2 \frac{e^{-\frac{\pi}{2}}}{\sinh \left(\frac{\pi}{2}\right)}+\frac{e^{\frac{\pi}{2}}\left(1-e^{-2 \pi}\right)}{2 \sinh ^{3}\left(\frac{\pi}{2}\right)}\right] \mathrm{B} .3\right)
$$

Now, using the value of the integral given in Eq. (B.3), we evaluate the following integrals found in Eq. (B.1) by integrating them by parts successively. 


$$
\begin{aligned}
& \int_{-\infty}^{\infty} d \zeta \zeta^{2} \operatorname{sech}^{2} \zeta e^{ \pm i \zeta}=\frac{\pi^{2}}{\sinh \frac{\pi}{2}}\left[ \pm\left(1 \mp \frac{\pi}{4}\right)+\frac{\left(1 \mp \frac{\pi}{2}\right) e^{\mp \frac{\pi}{2}}}{\sinh \frac{\pi}{2}}\right. \\
& \left. \pm \frac{\pi e^{ \pm \frac{\pi}{2}}\left(1-e^{\mp 2 \pi}\right)}{\sinh ^{3} \frac{\pi}{2}}\right] \\
& \int_{-\infty}^{\infty} d \zeta \zeta^{2} \operatorname{sech}^{2} \zeta \tanh \zeta e^{ \pm i \zeta}=\frac{i \pi}{\sinh \frac{\pi}{2}}\left[\left(\pi \mp 1 \mp \frac{\pi^{2}}{8}\right) \pm \frac{\pi\left(2 \mp \frac{\pi}{2}\right) e^{\mp \frac{\pi}{2}}}{2 \sinh \frac{\pi}{2}}\right. \\
& \left.-\frac{i \pi^{2} e^{ \pm \frac{\pi}{2}}\left(1-e^{\mp 2 \pi}\right)}{16 \sinh ^{3} \frac{\pi}{2}}\right] \text {. }
\end{aligned}
$$

On substituting Eqs. (B.4a) and (B.4b) in Eq. (B.1) we obtain

$$
\begin{aligned}
\Psi^{(1)}\left(\zeta, t_{0}\right)= & \frac{(1+v) i \pi^{3}}{16 \sinh \frac{\pi}{2}}\left[\frac{\cosh \frac{3 \pi}{2}}{\sinh ^{3} \frac{\pi}{2}}-\cosh \frac{\pi}{2}\left(\frac{4}{\sinh \frac{\pi}{2}}+1\right)\right] \operatorname{sech} \zeta \\
& +\frac{i}{2} \int_{-\infty}^{\infty} d k \frac{\left(1-k^{2}-2 i k \tanh \zeta\right)}{k\left(1+k^{2}\right)^{3}} e^{\frac{i\left(1+k^{2}\right) \alpha}{k}+i k \zeta}\left[\left\{k^{2}+b(1-b)\right.\right. \\
& \left.\times\left(1+k^{2}\right)^{2}\right\}\left(\operatorname{sech} \frac{\pi}{2}(\beta+1)+\operatorname{sech} \frac{\pi}{2}(\beta-1)\right)-\frac{\pi^{2}}{4} b(1-b) \\
& \left.\times\left(1+k^{2}\right)^{2} \operatorname{sech} \frac{\pi}{2} \beta\right]-\frac{i}{2} \int_{-\infty}^{\infty} d k \frac{\left(k-k^{3}-2 i k \tanh \zeta\right)}{\left(1+k^{2}\right)^{3}} \\
& \times e^{i k \zeta}\left(\operatorname{sech} \frac{\pi}{2}(1-k)+\operatorname{sech} \frac{\pi}{2}(1+k)\right) .
\end{aligned}
$$

Before evaluating the integrals in Eq.(B.5) we rewrite the same appropriately. We then evaluate the integrals in Eq. (B.5) one by one by finding the values of the residues at the pole $(k=i)$ at different orders. The residue for the integrand function $\left(\operatorname{sech} \frac{\pi}{2}(\beta+1)+\operatorname{sech} \frac{\pi}{2}(\beta-1)\right) \frac{k\left(1-k^{2}-2 i k \tanh \zeta\right)}{\left(1+k^{2}\right)^{3}} e^{\frac{i\left(1+k^{2}\right) \alpha}{k}+i k \zeta}$ at the pole $k=i$ of order three is found to be

$$
\left.\operatorname{Res}(k=i)=-\frac{\pi}{16}[2(2 b-1) \zeta+4(2 b-1) \alpha+1)\right] \operatorname{sech} \zeta
$$

Next, we find the residue for the function $e^{\frac{i\left(1+k^{2}\right) \alpha}{k}}+i k \zeta \frac{\left(1-k^{2}-2 i k \tanh \zeta\right)}{k\left(1+k^{2}\right) \cosh \beta \frac{\pi}{2}}$ at the simple poles $k=i$ and $k=\frac{(2 n+1)}{(1-v)}\left(-i \pm \sqrt{\frac{1-v^{2}}{(2 n+1)^{2}}-1}\right)$ ( which is out of contour). Further, at $k=0$ there is an essential singularity, the residue of which is not shown here due its unwieldy form. Thus the residue for the above function at the first order pole $k=i$ is given by

$$
\operatorname{Res}(k=i)=\frac{1}{\pi(1-2 b)^{2}}[2(2 b-1) \zeta+4(2 b-1) \alpha-1] \operatorname{sech} \zeta
$$


The residues for the function $\left(\operatorname{sech} \frac{\pi}{2}(k-1)+\operatorname{sech} \frac{\pi}{2}(k+1)\right) \frac{\left(k-k^{3}-2 i k^{2} \tanh \zeta\right)}{\left(1+k^{2}\right)^{3}} e^{i k \zeta}$ at the pole $k=i$ of order three and at the simple poles $k=i(2 n+1)+1$ and $k=i(2 n+1)-1$ are written as

$$
\begin{aligned}
\operatorname{Res}(k=i)= & \frac{\pi}{16}(2 \zeta-1) \operatorname{sech} \zeta, \\
\operatorname{Res}[k=i(2 n+1)+1]= & \frac{-2 i}{\pi} e^{(i-1) \zeta} \sum_{n=0} \frac{(-1)^{-n}[1+i(2 n+1)] e^{-2 n \zeta}}{\left[2-(2 n+1)^{2}+2 i(2 n+1)\right]^{3}} \\
& \times\left\{\left[(2 n+1)^{2}-2 i(2 n+1)\right]\right. \\
& -2 i \tanh \zeta[1+i(2 n+1)]\} \\
\operatorname{Res}[k=i(2 n+1)-1]= & \frac{2 i}{\pi} e^{-(1+i) \zeta} \sum_{n=0} \frac{(-1)^{-n}[-1+i(2 n+1)] e^{-2 n \zeta}}{\left[(2 n+1)^{2}-2+2 i(2 n+1)\right]^{3}} \\
& \times\left\{\left[(2 n+1)^{2}+2 i(2 n+1)\right]\right. \\
& -2 i \tanh \zeta[-1+i(2 n+1)]\} .
\end{aligned}
$$

It can be verified that the residue of the function $\left(\operatorname{sech} \frac{\pi}{2}(\beta+1)+\operatorname{sech} \frac{\pi}{2}(\beta-1)\right)$ $\frac{\left(1-k^{2}-2 i k \tanh \zeta\right)}{k\left(1+k^{2}\right)} e^{\frac{i\left(1+k^{2}\right) \alpha}{k}+i k \zeta}$ at $k=i$ vanishes. On summing up the values of the residues given in Eqs. (B.6), (B.7) and (B8) (dropping higher order terms in Eqs. (B.8b) and (B.8c) while adding) we obtain the following expression for $\psi^{(1)}\left(\zeta, t_{0}\right)$.

$$
\begin{aligned}
\Psi^{(1)}\left(\zeta, t_{0}\right) \approx & \frac{\pi^{2}}{4(1-2 b)^{2}} \operatorname{sech} \zeta\left[b(2 b-1) \zeta+\{b(2 \alpha+b-1)-\alpha\}+\frac{4}{122825}\right. \\
& \times\{(\cosh 2 \zeta-\sinh 2 \zeta)[25 \cosh 2 \zeta(523 \cos \zeta-1512 \sin \zeta)+289 \\
& (19 \cos \zeta+8 \sin \zeta)+15(766 \cos \zeta-2393 \sin \zeta) \sinh 2 \zeta]\}] . \quad(\text { B.9) }
\end{aligned}
$$

As residues due to the singularities in the plane except along the imaginary axis, lead to secular terms in the solutions, we take into account only singularities along the imaginary axis that is at $k=i$, dropping residues due to the other two singularities at $k=i(2 n+1)+1$ and $k=i(2 n+1)-1$. Thus the first order correction $\psi^{(1)}\left(\zeta, t_{0}\right)$ given in Eq.(B.9) finally becomes

$$
\Psi^{(1)}\left(\zeta, t_{0}\right) \approx \frac{\pi^{2}}{4(1-2 b)^{2}}[b(2 b-1) \zeta+\{b(2 \alpha+b-1)-\alpha\}] \operatorname{sech} \zeta
$$

\section{References}

[1] L. Stryer, Biochemistry. $4^{\text {th }}$ ed (W. H. Freeman and Company, New York, 1995).

[2] L. V. Yakushevich, Physica D 79 (1994) 77. 
[3] L. V. Yakushevich, Nonlinear Physics of DNA ( Wiley-VCH, Berlin, 2004).

[4] S. W. Englander, N. R. Kallenbanch, A. J. Heeger, J. A. Krumhansl and S. Litwin, Proc. Natl. Acad. Sci. U.S.A 77 (1980) 7222 .

[5] S. Yomosa, Phys. Rev. A 27 (1983) 2120.

[6] S. Yomosa, Phys. Rev. A 30 (1984) 474.

[7] J. Frenkel and T. Kontrova, J. Phys. (USSR) 1 (1939) 137.

[8] S. Takeno and S. Homma, Prog. Theor. Phys. 70 (1983) 308.

[9] S. Takeno and S. Homma, Prog. Theor. Phys. 72 (1984) 679.

[10] M. Peyrard and A. R. Bishop, Phys. Rev. Lett. 62 (1989) 2755.

[11] P. L. Christiansen, P. C. Lomdahl and V. Muto, Nonlinearity 4 (1991) 477.

[12] P. Jensen, M. V. Jaric and K. H. Bannenmann, Phys. Letts. A 95 (1983) 204.

[13] A. Khan, D. Bhaumik and B. Dutta-Roy, Bull. Math. Biol. 47 (1985) 783.

[14] V. K. Fedyanin and V. Lisy, Studia Biophys. 116 (1986) 65.

[15] R. V. Polozov and L. V. Yakushevich, J. Theor. Biol. 130 (1988) 423.

[16] J. A. Gonzalez and M. M. Landrove, Phys. Letts. A 292 (2002) 256.

[17] L. V. Yakushevich, Nanobiology 1 (1992) 343.

[18] G. Kalosakas, K. Q. Rasmussen and A. R. Bishop, Synthetic Metals, 141 (2004) 93.

[19] V. Muto, J. Halding, P. L. Christiansen and A. C. Scott, J. Biomol. Struc. Dyn. 5 (1988) 873.

[20] C. T. Zhang, Phys. Rev. A 40 (1989) 2148.

[21] M. Salerno, Phys. Rev. A 44 (1991) 5292.

[22] M. Salerno, Phys. Letts. A 167 (1992) 49.

[23] L. V. Yakushevich, A. V. Savin and L. I. Manevitch, Phys. Rev . E 66 (2002) 016614.

[24] A. Campa, Phys. Rev. E 63 (2001) 021901.

[25] K. Forinash, M. Peyrard and B. Malomed, Phys. Rev. E 49 (1994) 3400.

[26] J. A. D. Wattis, S. A. Harris, C.R. Grindon and C. A. Laughton, Phys. Rev. E 63 (2001) 061903.

[27] J. Cuevas, F. Palmero, J. F. R. Archilla and F. R. Romero, Phys. Letts. A 299 (2002) 221.

[28] S. Takeno, Phys. Letts. A 339 (2005) 352. 
[29] J. Ladik and J. Cizek, Int. J. Quantum Chem. 26 (1984) 955.

[30] E. Cubero, E. C. Sherer, F. J. Luque, M. Orozco and C. A. Laughton, J. Am. Chem. Soc. 121 (1999) 8653.

[31] O. M. Braun and Y. S. Kivshar, Phys. Rev. B 43 (1991) 1060.

[32] F. Zhang, Y. S. Kivshar and L. Vazquez, Phys. Rev. A 45 (1992) 6019.

[33] E. T. Kool, Annu. Rev. Biophys. Biomol. Struct. 30 (2001) 1.

[34] M. Hisakado and M. Wadati, J. Phys. Soc. Jpn. 64 (1995) 1098.

[35] R. Dandoloff and A. Saxena, J. Phys.: Condens. Matter 9 (1997) L667.

[36] M. J. Ablowitz, D. J. Kaup, A. C. Newell, and H. Segur, Stud. Appl. Math. 53 (1974) 249.

[37] D. J. Kaup, SIAM J. Appl. Math. 31 (1976) 12.

[38] G. L. Lamb, Elements of Soliton Theory (Wiley, New York, 1980).

[39] K. A. Gorshkov and L. A. Ostrovskii, Physica D 3 (1981) 428.

[40] D. J. Kaup, Phys. Rev. B 27 (1983) 6787.

[41] D. J. Kaup, Phys. Rev. B 29 (1984) 1072.

[42] J. P. Keener and D. W. McLaughlin, J. Math. Phys. 18 (1977) 2008.

[43] J. P. Keener and D. W. McLaughlin, Phys. Rev. A 16 (1977) 777.

[44] R. L. Herman, J. Phys. A 23 (1990) 1063.

[45] R. L. Herman, J. Phys. A 23 (1990) 2327.

[46] J. Yan and Y. Tang, Phys. Rev. E 54 (1996) 6816.

[47] Y. Tang and W. Wang, Phys. Rev. E 62 (2000) 8842.

[48] J. Yan, Y. Tang, G. Zhou and Z. Chen, Phys. Rev. E 58 (1998) 1064.

[49] H. Frauenfelder, Int. J. Quantum Chem. 35 (1989) 711.

[50] G. Kalosakas, K. O. Rasmussen, A. R. Bishop, C. H. Choi and A. Usheva, Europhys. Lett. 68 (1) (2004) 127.

[51] E. Kreyszig, Advanced Engineering Mathematics (John-Wiley, New York, 2002). 